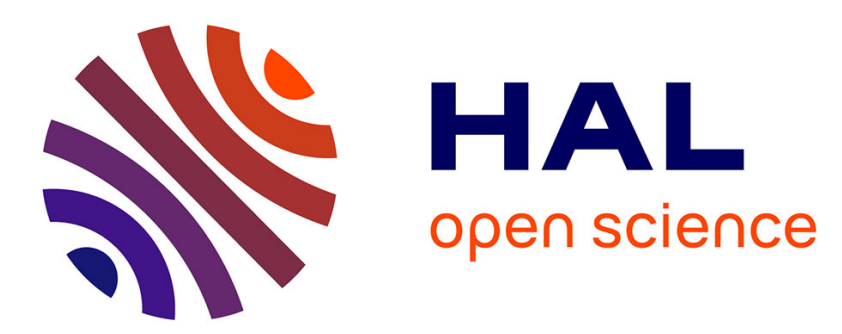

\title{
Residues on Affine Grassmannians
}

Mathieu Florence, Philippe Gille

\section{- To cite this version:}

Mathieu Florence, Philippe Gille. Residues on Affine Grassmannians. 2019. hal-02338391v3

\section{HAL Id: hal-02338391 \\ https://hal.science/hal-02338391v3}

Preprint submitted on 5 Feb 2021 (v3), last revised 16 Mar 2021 (v4)

HAL is a multi-disciplinary open access archive for the deposit and dissemination of scientific research documents, whether they are published or not. The documents may come from teaching and research institutions in France or abroad, or from public or private research centers.
L'archive ouverte pluridisciplinaire HAL, est destinée au dépôt et à la diffusion de documents scientifiques de niveau recherche, publiés ou non, émanant des établissements d'enseignement et de recherche français ou étrangers, des laboratoires publics ou privés. 


\title{
RESIDUES ON AFFINE GRASSMANNIANS
}

\author{
M. FLORENCE AND P. GILLE
}

\begin{abstract}
Given a linear group $G$ over a field $k$, we define a notion of index and residue of an element $g \in G(k((t)))$. The index $r(g)$ is a rational number and the residue a group homomorphism $\operatorname{res}(g): \mathbb{G}_{a}$ or $\mathbb{G}_{m} \rightarrow G$. This provides an alternative proof of Gabber's theorem stating that $G$ has no subgroups isomorphic to $\mathbb{G}_{a}$ or $\mathbb{G}_{m}$ iff $G(k[[t]])=G(k((t)))$. In the case of a reductive group, we offer an explicit connection with the theory of affine grassmannians.
\end{abstract}

Keywords: Group schemes, residues, affine grassmannians.

MSC 2000: 14M15, 20G35

\section{Contents}

1. Introduction 2

2. Indices 3

2.1. Notations and conventions 3

2.2. The ramification index 3

3. The residue 8

3.1. Left index, left residue 13

3.2. Extension to more affine group schemes $\quad 13$

3.3. Wound $A$-groups $\quad 13$

4. Residues for torsors 14

4.1. Definition 14

4.2. The wound case 14

4.3. The commutative case $\quad 15$

5. The case of fields, II $\quad 15$

5.1. Pseudo-complete varieties 16

5.2. Affine $k$-groups 16

5.3. Torsors over fields 17

6. Link with Gabber's compactifications 19

6.1. The statements 19

6.2. Refinement of Theorem $5.4 \quad 19$

7. Applications and examples 20

7.1. Applications 20

Date: February 5, 2021.

The authors are supported by the project ANR Geolie, ANR-15-CE 40-0012, (The French National Research Agency). 
7.2. Examples of $k$-groups such that $\operatorname{ker}\left(a_{G}\right) \neq 1 \quad 21$

8. A more advanced viewpoint 21

8.1. Automorphisms of Laurent series and pro-group schemes 22

8.2. Torsors and cocycles for Hochschild cohomology 22

8.3. Compararison with the elementary construction 23

9. Loop groups and affine Grassmannians 25

9.1. Ind-schemes 25

9.2. Ind-schemes, II 27

9.3. Case of a split reductive $k$-group 27

10. Appendix: descent 31

References $\quad 32$

\section{INTRODUCTION}

Let $k$ be a field and let $G$ be a linear algebraic $k$-group. Our goal is to associate in a quite elementary way to each element $g \in G(k((t))) \backslash G(k[[t]])$ an index $r(g) \in \mathbb{Q}_{\geq 0}$ and a non-trivial homomorphism $\operatorname{res}(g): \mathbb{G}_{a} \rightarrow G$ (resp. a non-trivial homomorphism $\operatorname{res}(g): \mathbb{G}_{m} \rightarrow G$ ) if $r(g)>0$ (resp. $r(g)=0$ ). If $k$ is of characteristic zero (resp. $p>0$ ), we show that the index $r(g)$ is integral (resp. belongs to $\mathbb{Z}_{(p)}$ ), see Corollary 3.5 .

This construction works actually over any ring $A$ with a closed subgroup scheme of $\mathrm{SL}_{N}$. This permits to recover results by Gabber [20] on the characterization of wound $k$-groups (i.e. $G$ does not contain $\mathbb{G}_{a}$ or $\mathbb{G}_{m}$ ) and also provides an extension in the group scheme setting. This extends also to points $x \in X(k((t))) \backslash X(k[[t]])$ for $X$ a $G$-torsor; this is a key ingredient in the proof of the following result.

Theorem 1.1. (see Theorem 5.4) Let $X$ be a $G$-torsor such that $X(k((t))) \neq \emptyset$. Then $X(k) \neq \emptyset$.

For reductive groups, this statement is due to Bruhat-Tits (see [15, I.1.2.1] or [18, prop. 5.5]). The generalization of that statement over a ring is known for $\mathrm{GL}_{n}$ and for tori according to recent results by Bouthier-Česnavičius [3, 2.1.17, 3.1.7]; we generalize it as well for wound closed subgroup schemes of $\mathrm{SL}_{N}$ (Cor. 4.2) and for $G$ commutative under further assumptions (Th. 4.3). It is an open question beyond those cases.

Already over a field it is an open question whether the statement does generalize to homogeneous spaces; this is the case in characteristic 0 according to results by the first author [13].

If $G$ is split reductive, we relate our construction of index and residue to the affine grassmannians $\mathcal{Q}_{G}$. The index provides a refinement of the stratification of the affine grasmannian $\mathcal{Q}_{G}$ of $G(\S 9.1)$. In particular we show that an element 
$g \in G(k((t))) \backslash G(k[[t]])$ has index 0 if and only if $g=g_{1} \mu(t) g_{2}$ where $g_{1} \in G(k)$, $g_{2} \in G(k[[t]])$ for some homomorphism $\mu: \mathbb{G}_{m} \rightarrow G$, see Proposition 9.9.

It is a pleasure to thank Ofer Gabber and Laurent Moret-Bailly for useful conversations. We thank also Simon Riche and Xinwen Zhu for their expertise on affine grassmannians and Alexis Bouthier for his reading. Finallly we would like to thank the reviewers for their helpful comments.

\section{INDICES}

2.1. Notations and conventions. If $r \in \mathbb{Q}^{\times}$, the notation $r=m / n$ means that $(m, n)=1$ with $n \geq 1$. This extends to $0=0 / 1$.

For each ring $A$ (commutative, unital), we denote by $A^{u}=A[u]$ the $\operatorname{ring}$ of $A$ polynomials in the indeterminate $u$. We denote by $A[[t]]$ the ring of power series and define $A((t))=A[[t]][x] /(1-t x)$. For each non-negative integer $n \geq 1$, we define $A\left[\left[t^{1 / n}\right]\right]=A[[t]][y] /\left(y^{n}-t\right)$ and $A\left(\left(t^{1 / n}\right)\right)=A[[t]][x, y] /\left(y^{n}-t, 1-x y\right)$. We have natural maps $A\left[\left[t^{1 / n}\right]\right] \rightarrow A\left[\left[t^{1 / m n}\right]\right]$ and $A\left(\left(t^{1 / n}\right)\right) \rightarrow A\left(\left(t^{1 / m n}\right)\right)$ for $m \geq 1$.

If $r=m / n \in \mathbb{Q}_{\geq 0}$, we put $A_{r}=A^{u}\left[\left[t^{1 / n}\right]\right]$ and $\mathcal{A}_{r}=A^{u}\left(\left(t^{1 / n}\right)\right)$. We have a specialization homomorphism $j: A_{r} \rightarrow A^{u}$.

For each $r=m / n \in \mathbb{Q}_{\geq 0}$, the assignment $t \rightarrow t\left(1+u t^{r}\right)$ defines ring homomorphisms $\sigma_{r}: A^{u}[[t]] \rightarrow A_{r}$; if $r>0$, its extends to $\sigma_{r}: A^{u}((t)) \rightarrow \mathcal{A}_{r}$.

Inverting $\lambda:=1+u$, we come now to analogues $\left.A^{u,+}=A[u][z] /(1-(1+u) z)\right)=$ $A\left[\lambda, \lambda^{-1}\right]$. We have the variants $A_{r}^{+}=A^{u,+}\left[\left[t^{1 / n}\right]\right] ; \mathcal{A}_{r}^{u,+}=A^{u,+}\left(\left(t^{1 / n}\right)\right), \sigma_{r}: A^{u,+}((t)) \rightarrow$ $\mathcal{A}_{r}^{+}, t \mapsto t\left(1+u t^{r}\right)$, and the specialization $j^{+}: A_{r}^{u,+} \rightarrow A^{u,+}$ for all $r \in \mathbb{Q}_{\geq 0}$.

2.2. The ramification index. Let $A$ be a ring and let $G$ be an affine $A$-group scheme equipped with a closed embedding $\rho: G \rightarrow \mathrm{SL}_{N, A}$.

Proposition 2.1. Let $g \in G(A((t))) \backslash G(A[[t]])$.

(1) The set

$$
\Sigma(g)=\left\{r \in \mathbb{Q}_{>0} \mid g^{-1} \sigma_{r}(g) \in G\left(A_{r}\right)\right\}
$$

is non-empty and let $r(g)$ be its lower bound in $\mathbb{R}$. Then $r(g) \in \mathbb{Q}_{\geq 0}$ and $\Sigma(g)=\mathbb{Q}_{>0} \cap[r(g),+\infty[$.

(2) Assume that $r(g)>0$. Then $j\left(g^{-1} \sigma_{r(g)}(g)\right)$ belongs to $G\left(A^{u}\right) \backslash G(A)$.

(3) Assume that $r(g)=0$. Then $g^{-1} \sigma_{0}(g) \in G\left(A^{u,+}[[t]]\right)$ and $j\left(g^{-1} \sigma_{0}(g)\right)$ belongs to $G\left(A^{u,+}\right) \backslash G(A)$.

(4) Assume that $r(g)=m / n>0$. Let $\sigma: A[[t]] \rightarrow A_{r(g)}$ be a homomorphism such that $\sigma(t)=t\left(1+u t^{r}+P_{2}(u) t^{m_{2} / n}+\ldots\right)$ with $m<m_{2}<m_{3}<\ldots<\ldots$ and $P_{i}(u) \in A^{u}$ for $i \geq 2$. Then $g^{-1} \sigma(g)$ belongs to $G\left(A_{r(g)}\right)$ and $j\left(g^{-1} \sigma(g)\right)=j\left(g^{-1} \sigma_{r}(g)\right)$.

Proof. (1) Clearly the statement reduces to the case of $\mathrm{SL}_{N}$. Our assumption implies that $g=t^{-d} \underline{g}$ with $d \geq 1$ and $\underline{g} \in \mathrm{M}_{N}(A[[t]]) \backslash t \mathrm{M}_{N}(A[[t]])$. The number $-d$ is called 
the gauge in $t$ of the matrix $g \in \mathrm{M}_{N}(A((t)))$ and is denoted ${ }^{1}$ by $V_{t}(g)$. It follows that $\operatorname{det}(\underline{g})=t^{N d}$. For $r \in \mathbb{Q}_{>0}$, we have

$$
g^{-1} \sigma_{r}(g)=\frac{t^{d}}{t^{d}\left(1+u t^{r}\right)^{d}} \underline{g}^{-1} \sigma_{r}(\underline{g})=\left(1+u t^{r}\right)^{-d} \underline{g}^{-1} \sigma_{r}(\underline{g}) .
$$

We write $\underline{g}=\left(P_{i, j}\right)_{i, j=1, . ., N}$ with $P_{i, j} \in A[[t]]$ and denote by $\Delta_{i, j} \in A[[t]]$ the minor of index $(i, j)$ of $\underline{g}$. We have $\underline{g}^{-1}=\left(t^{-N d} \Delta_{i, j}\right)_{i, j=1, . ., N}$ so that the $(i, j)$-coefficient $C_{i, j, r}$ of $\underline{g}^{-1} \sigma_{r}(\underline{g})$ is

$$
C_{i, j, r}=t^{-N d} \sum_{k=1}^{N} \Delta_{i, k}(t) P_{k, j}\left(t\left(1+u t^{r}\right)\right) \in A_{r} .
$$

When $u=0, C_{i, j, r}$ specializes on $\delta_{i, j}$ so that

$$
C_{i, j, r}=\delta_{i, j}+t^{-N d} \sum_{k=1}^{N} \Delta_{i, k}(t)\left(P_{k, j}\left(t\left(1+u t^{r}\right)\right)-P_{k, j}(t)\right) .
$$

We consider the identity

$$
\sum_{k=1}^{N} \Delta_{i, k}(t)\left(P_{k, j}(t(1+\epsilon))-P_{k, j}(t)\right)=\sum_{a \geq 0, b \geq 1} c_{i, j}^{a, b} t^{a} \epsilon^{b}
$$

with $c_{i, j}^{a, b} \in A$. Taking $\epsilon=u t^{r}$, we get

$$
C_{i, j, r}=\delta_{i, j}+t^{-N d} \sum_{a \geq 0, b \geq 1} c_{i, j}^{a, b} t^{a+r b} u^{b}
$$

We consider the $\operatorname{sets} \operatorname{supp}(i, j)=\left\{(a, b) \mid c_{i, j}^{a, b} \neq 0\right\}$ and $\operatorname{supp}(g)=\bigcup_{(i, j)} \operatorname{supp}(i, j)$.

Claim 2.2. $\operatorname{supp}(g) \neq \emptyset$.

If $\operatorname{supp}(g)=\emptyset$, then $\underline{g}=\sigma_{r}(\underline{g})$ and all coefficients of $g$ belong to $A$ which contradicts the fact $\operatorname{det}(\underline{g})=t^{N d}$. The Claim is established and enables us to define the function

$$
f_{g}(r)=\operatorname{Inf}\{-N d+a+r b \mid(a, b) \in \operatorname{supp}(g)\} .
$$

We have $f_{g}(r) \geq r+f_{g}(0)$ so that $f$ admits positive values and $\Sigma(g)$ is not empty. Since $1+u t^{r} \in A_{r}^{\times}$, the set $\Sigma(g)$ consists in the positive rational numbers $r$ such that $\underline{g}^{-1} \sigma_{r}(\underline{g})$ belongs to $\mathrm{GL}_{N}\left(A_{r}\right)$. We get that

$$
\Sigma(g)=\left\{r \in \mathbb{Q}_{>0} \mid f_{g}(r) \geq 0\right\} .
$$

\footnotetext{
${ }^{1}$ The gauge is not multiplicative but is surmultiplicative, i.e. $V_{t}\left(g_{1} g_{2}\right) \geq V_{t}\left(g_{1}\right)+V_{t}\left(g_{2}\right)$.
} 
By definition of $r(g)$, we have

$$
r(g)=\operatorname{Inf}\left\{r \in \mathbb{Q}_{>0} \mid f_{g}(r) \geq 0\right\} \in \mathbb{R}_{\geq 0} .
$$

If $f_{g}(0) \geq 0$, then $r(g)=0$. If $f_{g}(0)<0$, then there exists $a, b$ such that $-N d+a+r(g) b=0$ whence $r(g) \in \mathbb{Q}_{>0}$. In both cases, we have $\Sigma(g)=\mathbb{Q}_{>0} \cap[r(g),+\infty[$.

(2) Along the proof of (1), we have seen that then there exists $a, b$ such that $-N d+a+r b=0$ and $c_{i, j}^{a, b} \neq 0$. Formula (2.5) shows that $j\left(g^{-1} \sigma_{r}(g)\right) \notin \mathrm{SL}_{N}(A)$.

(3) Once again, it is enough to consider the case of $\mathrm{SL}_{N, A}$. We recall the notation $A_{1}=A^{u}[[t]]$ and $\mathcal{A}_{1}=\mathcal{A}^{u}((t))$. If $r(g)=0$, we have $a-N d \geq 0$ for each $a$ occurring in formula (2.5) (more precisely such that $c_{i, j}^{a, b} \neq 0$ for some $b$ ). The point is that the computation of (1) works also for $r=0$. It follows that

$$
\underline{g}^{-1} \sigma_{0}(\underline{g})-I_{N} \in u \mathrm{M}_{N}\left(A_{1}\right)
$$

so that $\underline{g}^{-1} \sigma_{0}(\underline{g}) \in \mathrm{M}_{N}\left(A_{1}\right)$. Taking into account the identity (2.5) for $r=0$, we get (2.6)

$$
g^{-1} \sigma_{0}(g)=\lambda^{-d} \underline{g}^{-1} \sigma_{0}(\underline{g})=\lambda^{-d}\left[I_{N}+u M_{0}(u)+t M_{1}(u)+\ldots\right] \in \mathrm{M}_{N}\left(A\left[\lambda, \lambda^{-1}\right][[t]]\right) .
$$

with $M_{i}(u) \in \mathrm{M}_{N}(A[u])$ for $i=1,2, \ldots$ We have $j\left(g^{-1} \sigma_{0}(g)\right)=\lambda^{-d}\left(I_{N}+u M_{0}(u)\right)$. Assume that $j\left(g^{-1} \sigma_{0}(g)\right)=M \in \mathrm{SL}_{N}(A)$. The formula (2.6) above reads

$$
g^{-1} \sigma_{0}(g)=M+\lambda^{-d}\left(t M_{1}(u)+t^{2} M_{2}(u)+\ldots\right) \in \mathrm{M}_{N}\left(A\left[\lambda, \lambda^{-1}\right][[t]]\right) .
$$

We consider the subring $B=(A[\lambda][[t]])\left[\lambda^{-1}\right]$ of $A\left[\lambda, \lambda^{-1}\right][[t]]$ and its analogue $\mathcal{B}=(A[\lambda]((t)))\left[\lambda^{-1}\right] \subset A\left[\lambda, \lambda^{-1}\right]((t))$. The map $\sigma_{0}: A((t)) \rightarrow A\left[\lambda, \lambda^{-1}\right]((t)), t \mapsto \lambda t$ factorizes through $\mathcal{B}$. It follows that the equation (2.7) holds in $\mathrm{M}_{N}(\mathcal{B})$, i.e.

$$
g^{-1} g(\lambda t)=M+\lambda^{-d}\left[t M_{1}(u)+t^{2} M_{2}(u)+\ldots\right] \in \mathrm{M}_{N}(\mathcal{B}) .
$$

In other words we have

$$
g^{-1} g(\lambda t)=M+\lambda^{-d}\left[t M_{1}(\lambda-1)+t^{2} M_{2}(\lambda-1)+\ldots\right] \in \mathrm{M}_{N}(\mathcal{B}) .
$$

The homomorphism $A[\lambda]((t)) \rightarrow A((t)), \sum_{i \geq-L} P_{i}(\lambda) t^{i} \mapsto \sum_{i \geq-L} P_{i}(t) t^{d i}$ extends uniquely to a homomorphism $\varphi: \mathcal{B} \rightarrow A((t))$. Specializing the equation (2.8) by $\varphi$ yields that $g^{-1}\left(t^{d}\right) g\left(t^{d+1}\right) \in \mathrm{M}_{N}(A[[t]])$ hence

$$
g\left(t^{1+d}\right)=g\left(t^{d}\right) Q \text { with } Q \in \mathrm{M}_{N}(A[[t]]) .
$$

It follows that $V_{t}\left(g\left(t^{1+d}\right)\right)=V_{t}\left(g\left(t^{d}\right) Q\right) \geq V_{t}\left(g\left(t^{d}\right)\right)+V_{t}(Q) \geq V_{t}\left(g\left(t^{d}\right)\right)$, so that $-d(d+1) \geq-d^{2}$, this is a contradiction. We conclude that $j\left(g^{-1} \sigma_{0}(g)\right) \notin \mathrm{SL}_{N}(A)$.

(4) We write $r=r(g)>0$ for short and continue to work with $\mathrm{SL}_{N, A}$. We denote by $\left(\widetilde{C}_{i, j}\right)$ the entries of $\underline{g}^{-1} \sigma(\underline{g})$. Taking $\epsilon=u t^{r}+P_{2}(u) t^{m_{2} / r}+\ldots$, we have 


$$
\begin{aligned}
\widetilde{C}_{i, j} & =\delta_{i, j}+t^{-N d} \sum_{a \geq 0, b \geq 1} c_{i, j}^{a, b} t^{a}\left(u t^{r}+P_{2}(u) t^{m_{2} / n}+\ldots\right)^{b} \\
& =C_{i, j, r}+t^{-N d} \sum_{a \geq 0, b \geq 1} c_{i, j}^{a, b} t^{a}\left(u^{b} t^{b r}+b u^{b-1} P_{2}(u) t^{\frac{(b-1) m+m_{2}}{n}}+\text { upper terms }\right) .
\end{aligned}
$$

For each $(a, b) \in \operatorname{supp}(i, j)$, we have $-N d+a+b r \geq 0$, so that $-N d+a+\frac{(b-1) m+m_{2}}{n}>0$. Since $\underline{g}^{-1} \sigma_{r}(\underline{g})$ belongs to $G\left(A_{r}\right)$, it follows that $\underline{g}^{-1} \sigma(\underline{g})$ belongs to $G\left(A_{r}\right)$ and so does $g^{-1} \sigma(g)$. Furthermore the above computation shows that $\underline{g}^{-1} \sigma(\underline{g})=\underline{g}^{-1} \sigma_{r}(\underline{g})$ in $\mathrm{M}_{N}\left(A_{r}\right)$ modulo $t^{\frac{m_{2}-m}{n}}$. Thus $j\left(g^{-1} \sigma(g)\right)=j\left(g^{-1} \sigma_{r}(g)\right)$.

Remark 2.3. If $A$ is a field, by inspection of the proof, we see that

$$
r(g)=\operatorname{Inf}\left\{r \in \mathbb{Q}_{>0} \mid g^{-1} \sigma_{r}(g) \in G(A(u)[[t]])\right\} .
$$

For later use, we record the following consequence of the proof of Proposition 2.1.

Lemma 2.4. The assumptions are those of Proposition 2.1. Let $M$ be a positive integer.

(1) Assume that $r=r(g)=\frac{m}{n}$. Then we have

$$
g^{-1} \sigma_{s}(g) \in \operatorname{ker}\left(G\left(A^{u}\left[\left[t^{1 / n}\right]\right]\right) \rightarrow G\left(A^{u}\left[t^{1 / n}\right] / t^{\frac{M}{n}}\right)\right)
$$

for all $s=\frac{u}{n}$ with $u \geq m+M$.

(2) If $r(g)=0$ we have

$$
g^{-1} \sigma_{s}(g) \in \operatorname{ker}\left(G\left(A^{u}[[t]]\right) \rightarrow G\left(A^{u}[t] / t^{M}\right)\right)
$$

for each integers $s \geq M$.

Proof. (1) The $(i, j)$-coefficient of $g^{-1} \sigma_{s}(g)$ reads

$$
D_{i, j, s}=\left(1+u t^{s}\right)^{-d}\left(\delta_{i, j}+t^{-N d} \sum_{a \geq 0, b \geq 1} c_{i, j}^{a, b} t^{a+s b} u^{b}\right) .
$$

We write $s-r=\frac{v}{n}$ with $v \geq M$ and get

$$
D_{i, j, s}=\left(1+u t^{s}\right)^{-d}\left(\delta_{i, j}+\sum_{a \geq 0, b \geq 1} c_{i, j}^{a, b} t^{-N d+a+r b} t^{\frac{v b}{n}} u^{b}\right) .
$$

For each non zero $c_{i, j}^{a, b}$, we have $-N d+a+r b \geq 0$ so that $D_{i, j, s}-\delta_{i, j} \in t^{\frac{v}{n}} A^{u}\left[\left[t^{\frac{1}{n}}\right]\right] \subseteq$ $t^{\frac{M}{n}} A^{u}\left[\left[t^{\frac{1}{n}}\right]\right]$.

(2) Let $(s, M)$ be a couple of integers satisfying $s \geq M \geq 1$. the $(i, j)$-coefficient of $g^{-1} \sigma_{s}(g)$ reads 


$$
D_{i, j, s}=\left(1+u t^{s}\right)^{-d}\left(\delta_{i, j}+\sum_{a \geq 0, b \geq 1} c_{i, j}^{a, b} t^{-N d+a} t^{b s} u^{b}\right) .
$$

For each non zero $c_{i, j}^{a, b}$, we have $-N d+a \geq 0$ so that $D_{i, j, s}-\delta_{i, j} \in t^{s} A^{u}[[t]] \subseteq$ $t^{M} A^{u}[[t]]$.

Definition 2.5. Let $g \in G(A((t)))$. If $g \notin G(A[[t]])$, we define the ramification index $r(g)$ as in Proposition 2.1. If $g \in G(A[[t]])$, we define $r(g)=-1$.

It is straightforward to check that the index does not depend of the choice of the representation $\rho$.

Lemma 2.6. We have

$$
\{g \in G(A((t))) \mid r(g) \leq 0\}=\left\{g \in G(A((t))) \mid g^{-1} g(\lambda t) \in G\left(A\left[\lambda, \lambda^{-1}\right][[t]]\right)\right\} .
$$

Proof. Let $g \in G(A((t)))$. If $g \in G(A[[t]])$, it is obvious that $g$ belongs to the righthand side. If $g \notin G(A[[t]])$ and $r(g) \leq 0$, we have $r(g)=0$ and $g$ belongs to the right-hand side according to Proposition 2.1.(3).

Conversely we assume that $g$ belongs to the right-hand side, that is $g^{-1} g(\lambda t) \in$ $G\left(A\left[\lambda, \lambda^{-1}\right][[t]]\right)$. We are given $r=m / n>0$. Since $1+u t^{r}$ is invertible in $A^{u}\left[\left[t^{1 / n}\right]\right]$, we can make $\lambda=1+u t^{r}$ so that $g^{-1} \sigma_{r}(g) \in G\left(A^{u}[[t]]\right)$. Since it holds for each rational $r>0$, we get that $r(g)=0$ by definition of the index.

Lemma 2.7. (1) The function $g \rightarrow r(g)$ is right $G(A[[t]])$-invariant (resp. left $G(A)$ invariant) and is insensible to any injective base change $A \hookrightarrow A^{\prime}$.

(2) Let $\phi: A \rightarrow B$ be a morphism of rings. Then $r\left(g_{B}\right) \leq r(g)$.

(3) Let $f: G \rightarrow H$ be a homomorphism between affine A-group schemes of finite type.

(i) We have $r(f(g)) \leq r(g)$.

(ii) If $A$ is integral and $f$ is proper, we have $r(f(g))=r(g)$.

(4) Let $G_{1}, G_{2}$ be affine $A$-group schemes of finite type and consider the A-group scheme $G_{1} \times_{A} G_{2}$. For $g_{i} \in G_{i}(A((t)))$ we have $r\left(g_{1}, g_{2}\right) \leq \operatorname{Inf}\left(r\left(g_{1}\right), r\left(g_{2}\right)\right)$.

(5) Let $d$ be a non-negative integer and consider the map $\phi_{d}: A((t)) \rightarrow A((T))$ defined by $\phi_{d}(t)=T^{d}$. We consider the map $\phi_{d, *}: G(A((t))) \rightarrow G(A((T)))$.

(i) If $d$ is not a zero divisor in $A$, we have $r\left(\phi_{d, *}(g)\right)=d r(g)$.

(ii) If $A$ is of characteristic $p>0$ and $d=p^{e}$, we have $r\left(\phi_{d}(g)\right)=r(g)$.

Proof. (1), (2) and (3).(i) readily follows of the definition of $r(g)$.

(3)(ii): We can replace $A$ by its fraction field by (1), allowing us to assume that $A$ is a field. Since $f: G \rightarrow H$ is assumed to be proper, the valuative criterion of 
properness yields $f^{-1}\left(H\left(A(u)\left[\left[t^{r}\right]\right]\right) \cap G\left(A(u)\left(\left(t^{r}\right)\right)\right)=G\left(A(u)\left[\left[t^{r}\right]\right]\right)\right.$ for each $r \in \mathbb{Q}_{>0}$. Then Remark 2.3 shows that $r(f(g))=r(g)$.

(4) follows from the definition of the index.

(5) We are given $r=m / n \in \mathbb{Q}_{>0}$. In case (i), the change $T \mapsto T\left(1+u T^{r}\right)$ induces $t=T^{d} \mapsto T^{d}\left(1+u T^{r}\right)^{d}=t\left(1+u d t^{\frac{m}{n d}}+\ldots\right)$. According to Proposition 2.1.(4), we get that $r(g)=\frac{r\left(\phi_{d, *}(g)\right)}{d}$.

In case (ii), we have $t=T^{p^{e}} \mapsto T^{p^{e}}\left(1+u T^{r}\right)^{p^{e}}=t\left(1+u^{p^{e}} t^{m / n}+\ldots\right)$. We get then $r\left(\phi_{d, *}(g)\right)=r(g)$.

Corollary 2.8. Assume that $A$ is integral and of characteristic $p>0$. Let $e$ be a non-negative integer and let $F_{e}: G \rightarrow G^{(e)}$ be the e-iterated Frobenius morphism [10, II.7.1.4]. Then for each $g \in G(A((t)))$, we have $r\left(F_{e}(g)\right)=r(g)$.

Proof. Since $F_{e}$ is proper, this follows of Lemma 2.7.(3).(ii).

\section{THE RESIDUE}

Let $A, G$ and $g \in G(A((t)))$ as in Proposition 2.1. If $r(g)>0$, we define the residue $\operatorname{res}(g)$ as the image of $g^{-1} \sigma_{r(g)}(g)$ by the homomorphism $j_{*}: G\left(A_{r}\right) \rightarrow G\left(A^{u}\right)=G(A[u])$. We see it as an $A-\operatorname{map} \operatorname{res}(g): \mathbb{G}_{a, A}=\operatorname{Spec}(A[u]) \rightarrow$ $\underline{G}$ and will use sometimes the notation $\operatorname{res}(g)(u)$.

If $r(g)=0$, we define the residue $\operatorname{res}(g)$ as the image of $g^{-1} \sigma_{0}(g)$ by the homomorphism $j_{*}: G\left(A_{0}^{+}\right) \rightarrow G\left(A^{u,+}\right)=G\left(A\left[u, \frac{1}{1+u}\right]\right)$. Putting $\lambda=1+u$, we have $A^{u,+}=A\left[\lambda, \lambda^{-1}\right]$ so that we see the residue as an $A$-map $\operatorname{res}(g): \mathbb{G}_{m, A}=$ $\operatorname{Spec}\left(A\left[\lambda, \lambda^{-1}\right]\right) \rightarrow G$. Similarly we use sometimes the notation $\operatorname{res}(g)(\lambda)$.

If $r(g)=-1$, i.e. $g \in G(A[[t]])$, we put $\operatorname{res}(g)=1 \in G\left(A^{u}\right)$. Again this does not depend of the choice of a representation.

Examples 3.1. (1) If $G=\mathbb{G}_{m, A}$ and $g=\frac{1}{t^{d}}$, we have

$$
g^{-1} \sigma_{0}(g)=(1+u)^{d}=\lambda^{d} .
$$

In this case we have $r(g)=0$ and $\operatorname{res}(g)(\lambda)=\lambda^{d}$.

(2) If $A=k[\epsilon]$ is the ring of dual numbers and $G=\mathbb{G}_{m, A}$, we consider the element $g=1+\frac{\epsilon}{t}$. Then $g^{-1} \sigma_{r}(g)=\left(1-\frac{\epsilon}{t}\right)\left(1+\frac{\epsilon}{t\left(1+u t^{r}\right)}\right)=1-\epsilon u t^{r-1}$ so that $r(g)=1$.

(3) If $G=\mathbb{G}_{a, A}$ and $g=\frac{1}{t^{d}}$ with $d \in \mathbb{Z}_{\geq 1}$ invertible in $A$, we have

$$
g^{-1} \sigma_{r}(g)=\frac{-1}{t^{d}}+\frac{1}{t^{d}\left(1+u t^{r}\right)^{d}}=\frac{-d u t^{r}+\ldots}{t^{d}\left(1+u t^{r}\right)^{d}}
$$

In this case we have $r(g)=d$ and $\operatorname{res}(g)(u)=-d u$. 
(4) If $G=\mathbb{G}_{a, A}$ and $g=\frac{1}{t^{p}}$ with $A$ of characteristic $p>0$ we have

$$
g^{-1} \sigma_{r}(g)=\frac{-1}{t^{p}}+\frac{1}{t^{p}\left(1+u t^{r}\right)^{p}}=-\frac{u^{p} t^{r p}}{t^{p}\left(1+u t^{r}\right)^{p}}
$$

In this case we have $r(g)=1$ and $\operatorname{res}(g)(u)=-u^{p}$.

Example 3.2. We consider the case $G=\mathrm{GL}_{2}$, and the element $g=\left(\begin{array}{cc}t^{a} & P(t) \\ 0 & t^{d}\end{array}\right)$ with $P(t) \in A[t]$ and $a, d \in \mathbb{Z}$. Putting $\epsilon=u t^{r}$, we have

$$
g^{-1} \sigma_{r}(g)=t^{-a-d}\left(\begin{array}{cc}
t^{d} & -P(t) \\
0 & t^{a}
\end{array}\right)\left(\begin{array}{cc}
t^{a}(1+\epsilon)^{a} & P(t(1+\epsilon)) \\
0 & t^{d}(1+\epsilon)^{d}
\end{array}\right)=\left(\begin{array}{cc}
(1+\epsilon)^{a} & f \\
0 & (1+\epsilon)^{d}
\end{array}\right)
$$

with

$$
f=t^{-a-d}\left(t^{d} P(t(1+\epsilon))-t^{d}(1+\epsilon)^{d} P(t)\right)=t^{-a}\left(P(t(1+\epsilon))-(1+\epsilon)^{d} P(t)\right) .
$$

(a) We take $a, d \geq 1, P(t)=1$ and assume than $d$ is invertible in $A$. In this case, $P(t(1+\epsilon))-(1+\epsilon)^{d} P(t)=1-\left(1+u t^{r}\right)^{d}$ so that

$$
g^{-1} \sigma_{r}(g)=\left(\begin{array}{cc}
\left(1+u t^{r}\right)^{a} & -d t^{-a+r} u+\ldots \\
0 & \left(1+u t^{r}\right)^{d}
\end{array}\right)
$$

It follows that $r(g)=a$ and that $\operatorname{res}(g)=\left(\begin{array}{cc}1 & -d u \\ 0 & 1\end{array}\right)$.

(b) Assume that $A$ is an $\mathbb{F}_{p}$-algebra and take $d=p^{s}(s \geq 1)$ and $P(t)=t^{p^{m s}}$ with $m \geq 2$. Then

$f=t^{-a}\left(t^{p^{m s}}\left(1+\epsilon^{p^{m s}}\right)-\left(1+\epsilon^{p^{s}}\right) t^{p^{m s}}\right)=t^{-a}\left(t^{p^{m s}}\left(u t^{r}\right)^{p^{m s}}-t^{p^{m s}}\left(u t^{r}\right)^{p^{s}}\right)=-t^{-a+p^{m s}+r p^{s}}(u)^{p^{s}}+\ldots$

If $a>p^{m s}$, we have $-a+p^{m s}+r p^{s}=0$ so that $r=\frac{a}{p^{s}}+p^{m(s-1)}$. In particular $r$ can belong in $\mathbb{Z}\left[\frac{1}{p}\right] \backslash \mathbb{Z}$.

(c) For the multiplicative indeterminate $\lambda$, we compute also

$$
g^{-1} g(\lambda t)=t^{-a-d}\left(\begin{array}{cc}
t^{d} & -P(t) \\
0 & t^{a}
\end{array}\right)\left(\begin{array}{cc}
\lambda^{a} t^{a} & P(\lambda t) \\
0 & \lambda^{d} t^{d}
\end{array}\right)=\left(\begin{array}{cc}
\lambda^{a} & f \\
0 & \lambda^{d}
\end{array}\right)
$$

with

$$
f=t^{-a-d}\left(t^{d} P(\lambda t)-\lambda^{d} t^{d} P(t)\right)=t^{-a}\left(P(\lambda t)-\lambda^{d} P(t)\right) .
$$

If $a \leq-1$, we have $r(g)=0$ and $\operatorname{res}(g)=\left(\begin{array}{cc}\lambda^{a} & 0 \\ 0 & \lambda^{d}\end{array}\right)$. Furthermore for $g^{\prime}=$ $g \operatorname{res}(g)\left(t^{-1}\right)$, we have

$$
g^{\prime-1} g^{\prime}(\lambda t)=\left(\begin{array}{cc}
t^{a} & 0 \\
0 & t^{d}
\end{array}\right)\left(\begin{array}{cc}
\lambda^{a} & f \\
0 & \lambda^{d}
\end{array}\right)\left(\begin{array}{cc}
\lambda^{-a} t^{-a} & 0 \\
0 & \lambda^{-d} t^{-d}
\end{array}\right)=\left(\begin{array}{cc}
1 & f_{1} \\
0 & 1
\end{array}\right)
$$


with $f_{1}=t^{a-d} \lambda^{-d} f=\lambda^{-d} t^{-d}\left(P(\lambda t)-\lambda^{d} P(t)\right)$. For $a=-1, d=1$ and $P(t)=1$, we see that $g^{\prime-1} g^{\prime}(\lambda t)$ does not belong in $\mathrm{GL}_{2}\left(A\left[\lambda, \lambda^{-1}\right][[t]]\right)$.

Similarly for $g^{\prime \prime}=\operatorname{res}(g)\left(t^{-1}\right) g$, we have

$$
g^{\prime \prime-1} g^{\prime \prime}(\lambda t)=t^{-a-d}\left(\begin{array}{cc}
t^{d} & -P(t) \\
0 & t^{a}
\end{array}\right)\left(\begin{array}{cc}
\lambda^{-a} & 0 \\
0 & \lambda^{-d}
\end{array}\right)\left(\begin{array}{cc}
\lambda^{a} t^{a} & P(\lambda t) \\
0 & \lambda^{d} t^{d}
\end{array}\right)=\left(\begin{array}{cc}
1 & f_{2} \\
0 & 1
\end{array}\right)
$$

with $f_{2}=t^{-a}\left[\lambda^{-a} P(\lambda t)-P(t)\right]$. So for $a \leq-1$, we see that $g^{\prime \prime-1} g^{\prime \prime}(\lambda t)$ belongs to $\mathrm{GL}_{2}(A[[t]])$.

For a group $\Gamma$, we recall the notation ${ }^{\sigma} \tau=\sigma \tau \sigma^{-1}$ and $\tau^{\sigma}=\sigma^{-1} \tau \sigma$ for $\sigma, \tau \in \Gamma$.

Lemma 3.3. Let $g \in G(A((t)))$.

(1) Let $g_{1} \in G(A)$ and $g_{2} \in G(A[[t]])$. Then $\operatorname{res}\left(g_{1} g g_{2}\right)=\operatorname{res}(g)^{\overline{g_{2}}}$ where $\overline{g_{2}}$ stands for the specialization of $g_{2}$ in $G(A)$. In particular we have $\operatorname{res}\left({ }^{g_{1}} g\right)=\bar{g}_{1} \operatorname{res}(g)$.

(2) Let $A \rightarrow A^{\prime}$ be a base change such that $r(g)=r\left(g_{A^{\prime}((t))}\right)$ (it holds for example when $A$ injects in $\left.A^{\prime}\right)$. Then $\operatorname{res}\left(g_{A^{\prime}}\right)=\operatorname{res}(g)_{A^{\prime}}$.

(3) Assume that $A$ is integral and let $f: G \rightarrow H$ be a proper homomorphism between affine $A$-group schemes of finite type. We have $\operatorname{res}(f(g))=\operatorname{res}(g)$.

(4) Let $d$ be a non-negative integer and consider the map $\phi_{d}: A((t)) \rightarrow A((T))$ defined by $\phi_{d}(t)=T^{d}$. We consider the map $\phi_{d, *}: G(A((t))) \rightarrow G(A((T)))$.

(i) If $d$ is not a zero divisor in $A$, we have $\operatorname{res}\left(\phi_{d, *}(g)\right)(u)=\operatorname{res}(g)(d u)$ if $r>0$, or $\operatorname{res}\left(\phi_{d, *}(g)\right)(\lambda)=\operatorname{res}(g)\left(\lambda^{d}\right)$ if $r=0$.

(ii) If $A$ is of characteristic $p>0$ and $d=p^{e}$, we have $\operatorname{res}\left(\phi_{d}(g)\right)=\operatorname{res}(g)\left(u^{p^{e}}\right)$.

Proof. We write $r=r(g)=m / n$.

(1) Since $\sigma_{r}\left(g_{1}\right)=g_{1}$, we have $\left(g_{1} g g_{2}\right)^{-1} \sigma_{r}\left(g_{1} g g_{2}\right)=g_{2}^{-1}\left(g^{-1} \sigma_{r}(g)\right) \sigma_{r}\left(g_{2}\right)=g_{2}^{-1}\left(g^{-1} \sigma_{r}(g)\right) \sigma_{r}\left(g_{2}\right)$. When we specialize at $t=0$, we get $\operatorname{res}\left(g_{1} g g_{2}\right)=\operatorname{res}(g)^{\overline{g_{2}}}$. Assertions (2) and (3) follow of Lemma 2.7 .

(4) We continue the proof of Lemma 2.7.(5). We have four cases to verify.

Case (i), $r>0$. We have $r\left(\phi_{d, *}(g)\right)=d r(g)=\frac{d m}{n_{m}}$. The change $T \mapsto T\left(1+u T^{r}\right)$ induces $t=T^{d} \mapsto T^{d}\left(1+u T^{r}\right)^{d}=\tau(t)=t\left(1+d u t^{\frac{m}{n d}}+\ldots\right)$. It follows that

$$
\phi_{d, *}\left(g^{-1} \tau(g)\right)=\phi_{d, *}(g)^{-1} \sigma_{T, \frac{m}{n d}}\left(\phi_{d, *}(g)\right) \in G\left(A^{u}[[T]]\right)
$$

Proposition 2.1.(4) yields that $j\left(g^{-1} \tau(g)\right)=j\left(g^{-1} \sigma_{t, r}(g)\right)=d \operatorname{res}(g) \in G\left(A^{u}\right)$.

Case (i), $r=0$. The change $T \mapsto \lambda T$ induces $t=T^{d} \mapsto \lambda^{d} t$. It follows that

$$
\phi_{d, *}\left(g^{-1} g(\lambda t)\right)=\phi_{d, *}(g)^{-1} g\left(\lambda^{d} T\right) \operatorname{res}(g)\left(\lambda^{d}\right)(1+\epsilon) \in G\left(A\left[\lambda^{ \pm 1}[[T]]\right)\right.
$$

with $j(1+\epsilon)=1$. We conclude that $\operatorname{res}\left(\phi_{d, *}(g)\right)(\lambda)=\operatorname{res}(g)\left(\lambda^{d}\right)$. 
Case (ii), $r>0$. We have $r\left(\phi_{d, *}(g)\right)=r(g)$ and consider the base change $t=T^{p^{e}} \mapsto$ $T^{p^{e}}\left(1+u T^{r}\right)^{p^{e}}=\tau^{\prime}(t)=t\left(1+u^{p^{e}} t^{m / n}+\ldots\right)$. It follows that

$$
\phi_{d, *}\left(g^{-1} \tau^{\prime}(g)\right)=\phi_{d, *}(g)^{-1} \sigma_{T, r}\left(\phi_{d, *}(g)\right) \in G\left(A^{u}[[T]]\right) .
$$

We put $v=u^{p^{e}}$ and consider $\sigma_{r}^{v}: A^{v}[[t]] \rightarrow A^{v}\left[\left[t^{1 / n}\right]\right]$. By using Proposition 2.1.(4) and the functoriality of the construction $A^{v} \rightarrow A^{u}$, we have $j\left(g^{-1} \tau^{\prime}(g)\right)=j\left(g^{-1} \sigma_{r}^{v}(g)\right)=$ $\operatorname{res}(g)(v)=\operatorname{res}(g)\left(u^{p^{e}}\right) \in G\left(A^{u}\right)$. By specializing formula (3.1) at $T=0$, we get $\operatorname{res}\left(\phi_{d, *}(g)\right)(u)=\operatorname{res}(g)\left(u^{p^{e}}\right)$.

Case (ii), $r=0$. It is similar.

Theorem 3.4. (1) If $r(g)>0$, then $\operatorname{res}(g)$ is non-trivial homomorphism $\mathbb{G}_{a, A} \rightarrow G$. (2) If $r(g)=0$, then $\operatorname{res}(g)$ is a non-trivial homomorphism $\mathbb{G}_{m, A} \rightarrow G$.

Remark 3.0.1. In the case of an integral index, we provide an alternative proof for the homomorphism part of (1) and (2) in Remark 8.3.1.

Proof. We can continue to work with $\mathrm{SL}_{N}$. We write $r=r(g)=m / n$.

(1) We assume firstly that $n$ is invertible in $A$. By developing the serie $\left(1+u t^{r}\right)^{1 / n}$ in $A_{r}$, we can extend $\sigma_{r}: A^{u}[[t]] \rightarrow A_{r}$ to $\widetilde{\sigma}_{r}: A_{r} \rightarrow A_{r}$. The trick is to use the rings $A^{v_{1}, v_{2}}=A\left[v_{1}, v_{2}\right], A^{v_{1}, v_{2}}[[t]]$ and $A^{v_{1}, v_{2}}\left[\left[t^{1 / n}\right]\right]$ and to define morphisms $\tau_{i}: A^{v_{1}, v_{2}}[[t]] \rightarrow$ $A^{v_{1}, v_{2}}\left[\left[t^{1 / n}\right]\right](i=1,2,3)$ by $t \mapsto t\left(1+v_{1} t^{r}\right), t\left(1+v_{2} t^{r}\right), t\left(1+\left(v_{1}+v_{2}\right) t^{r}\right)$ respectively. These morphisms extend to morphisms $\widetilde{\tau}_{i}: A^{v_{1}, v_{2}}\left[\left[t^{1 / n}\right]\right] \rightarrow A^{v_{1}, v_{2}}\left[\left[t^{1 / n}\right]\right]$ for $i=1,2,3$. We have the cocycle relation

$$
g^{-1}\left(\widetilde{\tau}_{1} \widetilde{\tau}_{2}\right)(g)=g^{-1} \widetilde{\tau}_{1}(g) \widetilde{\tau}_{1}\left(g^{-1} \widetilde{\tau}_{2}(g)\right)
$$

inside $G\left(A^{v_{1}, v_{2}}\left(\left(t^{1 / n}\right)\right)\right)$. By using functoriality properties (Lemmas 2.7.(1) and 3.3.(2)) we have $g^{-1} \widetilde{\tau}_{i}(g) \in G\left(A^{v_{1}, v_{2}}\left[\left[t^{1 / n}\right]\right]\right)$ with specialization $\operatorname{res}(g)\left(v_{i}\right)$. It follows that

$$
g^{-1}\left(\widetilde{\tau}_{1} \widetilde{\tau}_{2}\right)(g)=\operatorname{res}(g)\left(v_{1}\right) \operatorname{res}(g)\left(v_{2}\right)
$$

inside $G\left(A^{v_{1}, v_{2}}\left[\left[t^{1 / n}\right]\right]\right)$ modulo the kernel of $G\left(A^{v_{1}, v_{2}}\left[\left[t^{1 / n}\right]\right]\right) \rightarrow G\left(A^{v_{1}, v_{2}}\right)$. On the other hand $\widetilde{\tau}_{1} \widetilde{\tau}_{2}(t)=t\left(1+\left(v_{1}+v_{2}\right) t^{r}+\right.$ upper terms $)$. Proposition 2.1.(3) applied to the ring $A\left[v_{1}\right]$ and $u=v_{1}+v_{2}$ shows that $g^{-1}\left(\widetilde{\tau}_{1} \widetilde{\tau}_{2}\right)(g)=\operatorname{res}(g)\left(v_{1}+v_{2}\right)$ inside $G\left(A^{v_{1}, v_{2}}\left[\left[t^{1 / n}\right]\right]\right)$ modulo the kernel of $G\left(A^{v_{1}, v_{2}}\left[\left[t^{1 / n}\right]\right]\right) \rightarrow G\left(A^{v_{1}, v_{2}}\right)$. We conclude that $\operatorname{res}(g)\left(v_{1}+v_{2}\right)=\operatorname{res}(g)\left(v_{1}\right) \times \operatorname{res}(g)\left(v_{2}\right)$. Thus res $(g)$ is an $A$-group homomorphism.

We explain now the refinement to the case $n=q n^{\prime}$ when $A$ is of characteristic $p>0$ and $\left(n^{\prime}, p\right)=1$ and $q=p^{e}$. We consider $A^{u}[[t]] \stackrel{\sigma_{r}}{\rightarrow} A^{u}\left[\left[t^{1 / n}\right]\right] \rightarrow A\left[u^{1 / q}\right]\left[\left[t^{1 / n q}\right]\right]$ to $\widetilde{\sigma}_{r}: A^{u}\left[\left[t^{1 / n}\right]\right] \rightarrow A\left[u^{1 / q}\right]\left[\left[t^{1 / n q}\right]\right]$ by mapping $t^{1 / n}$ to the series $\left(1+u^{1 / q} t^{r / q}\right)^{1 / n^{\prime}}$.

We extend then similarly the morphisms $A^{v_{1}^{q}, v_{2}^{q}}[[t]] \stackrel{\tau_{i}}{\rightarrow} A^{v_{1}^{q}, v_{2}^{q}}\left[\left[t^{1 / n}\right]\right] \rightarrow A^{v_{1}, v_{2}}\left[\left[t^{1 / n q}\right]\right]$ $(i=1,2,3)$ defined by $t \mapsto t\left(1+v_{1}^{q} t^{r}\right), t\left(1+v_{2}^{q} t^{r}\right), t\left(1+\left(v_{1}^{q}+v_{2}^{q}\right) t^{r}\right)$ in $\widetilde{\tau}_{i}: A^{v_{1}^{q}, v_{2}^{q}}\left[\left[t^{1 / n}\right]\right] \rightarrow$ 
$A^{v_{1}, v_{2}}\left[\left[t^{1 / n q}\right]\right]$ for $i=1,2,3$. The cocycle condition reads then

$$
g^{-1}\left(\widetilde{\tau}_{1} \tau_{2}\right)(g)=g^{-1} \tau_{1}(g) \widetilde{\tau}_{1}\left(g^{-1} \tau_{2}(g)\right)
$$

The same method yields $\operatorname{res}(g)\left(v_{1}^{q}+v_{2}^{q}\right)=\operatorname{res}(g)\left(v_{1}^{q}\right) \times \operatorname{res}(g)\left(v_{2}^{q}\right)$. Thus res $(g)$ is an A-group morphism.

(2) We have seen that $\operatorname{res}(g) \in G\left(A\left[\lambda^{ \pm 1}\right]\right) \backslash G(A)$ in Lemma 3.3.(4). We consider the ring $A\left[\lambda_{1}^{ \pm 1}, \lambda_{2}^{ \pm 1}\right]$ and the $A\left[\lambda_{1}, \lambda_{2}\right]$-automorphisms $\rho_{i}$ of $A\left[\lambda_{1}, \lambda_{2}\right]((t))\left[\lambda_{1}^{-1}, \lambda_{2}^{-1}\right]$ defined respectively by $\rho_{1}(t)=\lambda_{1} t, \rho_{2}(t)=\lambda_{2} t$, and $\rho_{3}(t)=\lambda_{1} \lambda_{2} t$. Since $\rho_{3}=\rho_{2} \circ \rho_{1}$, the cocycle relation $g^{-1}\left(\rho_{1} \rho_{2}\right)(g)=g^{-1} \rho_{1}(g) \rho_{1}\left(g^{-1} \rho_{2}(g)\right)$ in $G\left(A\left[\lambda_{1}, \lambda_{2}\right]((t))\left[\lambda_{1}^{-1}, \lambda_{2}^{-1}\right]\right)$ yields $\operatorname{res}(g)\left(\lambda_{1} \lambda_{2}\right)=\operatorname{res}(g)\left(\lambda_{1}\right) \operatorname{res}(g)\left(\lambda_{2}\right)$ as desired.

This provides some control on the indices in the integral case.

Corollary 3.5. Assume that $A$ is integral. Let $d \geq 1$ be an integer such that $t^{d} g \in$ $M_{N}(A[[t]])$.

(1) If $A$ contains $\mathbb{Z}$, then $r(g) \in \mathbb{Z}$ and $r(g) \leq N d$.

(2) If $A$ is a $\mathbb{F}_{p}$-algebra for a prime $p$, then $r(g) \in \mathbb{Z}\left[\frac{1}{p}\right]$ and there exists $s \geq 0$ such that $p^{s} r(g) \in \mathbb{Z}$ and $p^{s} r(g) \leq N d$.

Proof. (1) and (2). If $r(g)=0$ the statements are clear so that we can assume that $r(g)>0$. We are allowed to replace $A$ by its fraction field $F$ according to Lemma 3.3.(2). We use now the decomposition $\mathrm{SL}_{N}(F((t)))=B_{N}(F((t))) \mathrm{SL}_{N}(F[[t]])$ where $B_{N}$ stands for the $F$-subgroup of upper triangular matrices [4, 4.4.3]. Lemma 3.3.(1) permits to assume that $g \in B_{N}(F((t)))$. Coming back in the proof of Proposition 2.1, we consider the coefficients of $g^{-1} \sigma_{r}(g)$

$$
D_{i, j, r}=\left(1+u t^{r}\right)^{-d}\left(\delta_{i, j}+t^{-N d} \sum_{a \geq 0, b \geq 1} c_{i, j}^{a, b} t^{a+r b} u^{b}\right) .
$$

We have $D_{i, j, r}=0$ if $j<i$. We consider the non-empty set

$$
\Upsilon(g)=\left\{(i, j, a, b) \mid-N d+a+r b=0 \text { and } c_{i, j}^{a, b} \neq 0\right\} .
$$

It follows that the $(i, j)$-entry of $\operatorname{res}(g) \in B_{N}(k[u])$ is

$$
c_{i, j}=\delta_{i, j}+\sum_{(i, j, a, b) \in \Upsilon(g)} c_{i, j}^{a, b} u^{b}
$$

It follows that $c_{i, i}=1$ for each $i=1, . ., N$, that is $\operatorname{res}(g) \in U_{N}(k[u])$ where $U_{N}$ stands for the unipotent radical of $B_{N}$. Let $(i, j, a, b)$ in $\Upsilon(g)$ such that $i+j$ is minimal. Since $\operatorname{res}(g)$ is a group homomorphism, it follows that $u \mapsto c_{i, j}^{a, b} u^{b}$ is a group homomorphism. Case of characteristic zero. In this case we have $b=1$. The equation $-N d+a+r b=0$ yields that $r \in \mathbb{Z}$ and that $r=N d-a \leq N d$.

Case of characteristic $p$. It follows that $b$ is a $p$-power, i.e. $b=p^{s}$. Thus $r \in \mathbb{Z}\left[\frac{1}{p}\right]$ and $p^{s} r=N d-a \leq N d$. 
Remark 3.6. Corollary 3.5.(1) is strenghtened by Lemma 9.1.(1) which shows that $r(g) \leq N d$ without any condition on $A$.

3.1. Left index, left residue. Given $g \in G(A((t)))$, we define the left index and left residue by $r^{L}(g)=r\left(g^{-1}\right)$ and $\operatorname{res}^{L}(g)=\operatorname{res}\left(g^{-1}\right)$. If $G$ is commutative, we have $r^{L}(g)=r(g)$ and $\operatorname{res}^{L}(g)=-\operatorname{res}(g)$. This breaks in the non-commutative case.

Example 3.7. In $\mathrm{GL}_{2}(k((t)))$, we take $g=\left(\begin{array}{ll}t & 1 \\ 0 & t\end{array}\right)$. We have seen that $r(g)=1$. We have $g^{-1}=\left(\begin{array}{cc}t^{-1} & -t^{2} \\ 0 & t^{-1}\end{array}\right)$ and

$g \sigma_{r}\left(g^{-1}\right)=\left(\begin{array}{ll}t & 1 \\ 0 & t\end{array}\right)\left(\begin{array}{cc}t^{-1}\left(1-u t^{r}\right)^{-1} & -t^{-2}\left(1+u t^{r}\right)^{-2} \\ 0 & t^{-1}\left(1-u t^{r}\right)^{-1}\end{array}\right)=\left(\begin{array}{cc}\left(1-u t^{r}\right)^{-1} & 0 \\ 0 & \left(1+u t^{r}\right)^{-1}\end{array}\right)$

so that $r\left(g^{-1}\right)=0$.

3.2. Extension to more affine group schemes. Lemma 2.7.(1) implies in particular that the index (and residue) is insensible to a faithfully flat extension $A \rightarrow A^{\prime}$ of rings. Descent theory implies that their definition extend to group schemes admitting a closed embedding in $\mathrm{SL}_{N}$ after a faithfully flat extension. This applies in particular to group schemes of multiplicative type (finitely presented) and to reductive group schemes.

From now on, we may then assume that $G$ is a closed $A$-subgroup of $\mathrm{SL}_{N}$ or that $G$ is affine of finite presentation and there exists a faithfully flat extension $A \rightarrow A^{\prime}$ such that $G_{A^{\prime}}$ is a closed $A^{\prime}$-subgroup of some $\mathrm{SL}_{N^{\prime}, A^{\prime}}$.

\subsection{Wound $A$-groups.}

Corollary 3.8. The following conditions are equivalent:

(i) $G(A[[t]])=G(A((t)))$;

(ii) $G(A)=G\left(A\left[\lambda, \lambda^{-1}\right]\right)$;

(iii) $\operatorname{Hom}_{A-g r}\left(\mathbb{G}_{a}, G\right)=0$ and $\operatorname{Hom}_{A-g r}\left(\mathbb{G}_{m}, G\right)=1$.

Proof. $(i) \Longrightarrow($ ii $)$. We have $G(A[[t]])=G(A((t)))$ and similarly $G\left(A\left[\left[t^{-1}\right]\right]\right)=$ $G\left(A\left(\left(t^{-1}\right)\right)\right)$. If follows that $G\left(\mathbb{P}_{A}^{1}\right)=G\left(A\left[\lambda^{ \pm 1}\right]\right)$. Since $G$ is affine of finite type over $A$, we conclude that $G(A)=G\left(A\left[\lambda^{ \pm 1}\right]\right)$.

$($ ii $) \Longrightarrow($ iii $)$. Let $h: \mathbb{G}_{a} \rightarrow G$ be an $A$-homomorphism. Our assumption provides an element $g \in G(A)$ such that $h(u)=g$. Since $h(0)=1$, we conclude that $h$ is trivial. Similarly we have $\operatorname{Hom}_{A-g r}\left(\mathbb{G}_{m}, G\right)=1$.

$($ iii $) \Longrightarrow(i)$. This follows of Theorem 3.4.

Remark 3.9. If $A=F$ is a field, Corollary 3.8 was known in the reductive case as the Bruhat-Tits-Rousseau's theorem [21] (or [18, prop. 3.5]) and the general case is a consequence of Gabber's compactifications, this will be discussed in $\S 6$. 
Definition 3.10. If the affine $A$-group scheme $G$ of finite type satisfies the conditions of Corollary 3.8, we say that $G$ is $A$-wound ( $A$-ployé in French).

Our definition extends over any ring the case of wound algebraic groups over a field $[8,5.1]$.

\section{RESIDUES FOR TORSORS}

\subsection{Definition.}

Proposition 4.1. Let $X$ be a G-torsor over $A$. Let $x \in X(A((t))) \backslash X(A[[t]])$. For each $r \in \mathbb{Q}_{\geq 0}$, we denote by $g_{r}(x)$ the unique element of $G\left(\mathcal{A}_{r}\right)$ such that $\sigma_{r}(x)=x \cdot g_{r}(x)$.

(1) The set

$$
\Sigma(x)=\left\{r \in \mathbb{Q}_{>0} \mid g_{x}(r) \in G\left(A_{r}\right)\right\}
$$

is non-empty and let $r(x)$ be its lower bound in $\mathbb{R}$. Then $r(x) \in \mathbb{Q}_{\geq 0}$ and $\Sigma(x)=\mathbb{Q}_{>0} \cap[r(g),+\infty[$.

(2) If $r(x)>0$, then $j\left(g_{r(x)}(x)\right) \in G\left(A^{u}\right)$ is a non-trivial homomorphism $\operatorname{res}(x): \mathbb{G}_{a} \rightarrow G$.

(3) If $r(x)=0$, then $j\left(g_{r(x)}(x)\right) \in G\left(A^{u,+}\right)$ is a non-trivial homomorphism $\operatorname{res}(x): \mathbb{G}_{m} \rightarrow G$.

Proof. In the case of a trivial $G$-torsor, the result follows of Proposition 2.1 and of Theorem 3.4. We shall reduce to this case. Let $B$ a flat cover of $A$ such that $X_{B} \cong G_{B}$. Since $A$ embeds in $B$, we have $\Sigma(x)=\Sigma\left(x_{B}\right)$ so for (1) we are reduced to the case of $X_{B}$. The statements (2) and (3) follow by descent from $B$ to $A$.

Of course this extension of the residue to torsors satisfy the same functorialities as the residue for group schemes.

\subsection{The wound case.}

Corollary 4.2. We assume that $G$ is $A$-wound. Let $X$ be a $G$-torsor over $A$. Then $X$ is trivial if and only if $X \times_{A} A((t))$ is trivial.

Proof. The direct implication is obvious. We assume that $X \times_{A} A((t))$ is trivial, that is $X(A((t))) \neq \emptyset$. If $X(A[[t]]) \neq \emptyset$, then $X(A) \neq \emptyset$ and $X$ is a trivial $G$-torsor. We can assume then that $X(A[[t]])=\emptyset$. We pick $x \in X(A((t)))$ and Proposition 4.1 provides an element res $(x)$ which is a non-trivial morphism $\mathbb{G}_{a} \rightarrow G$ or a non-trivial morphism $\mathbb{G}_{m} \rightarrow G$. This contradicts our assumption. 


\subsection{The commutative case.}

Theorem 4.3. We assume that $A$ is an integral $\mathbb{Q}$-algebra, and that the $A$-group scheme $G$ is commutative. Let $X$ be a $G$-torsor over $A$. Then $X$ is trivial if and only if $X \times_{A} A((t))$ is trivial.

Proof. We assume that the $G$-torsor $X \times_{A} A((t))$ is trivial, that is $X(A((t))) \neq \emptyset$. If $X(A)=\emptyset$, then the indices of points of $X(A((t)))$ are all non-negative integers. Let $r$ be the minimal value of those indices and consider a point $x \in X(A((t)))$ such that $r(x)=r$.

Additive case: $r \geq 1$. We consider the point $y=x \cdot \operatorname{res}(x)\left(-\frac{1}{r t^{r}}\right)$. According to Example 3.1.(2), we have

$$
-\frac{1}{t^{r}}+\sigma_{r}\left(\frac{1}{t^{r}}\right)=r u+r \epsilon
$$

with $\epsilon \in t A^{u}[[t]]$. Since $G$ is commutative, we have

$$
\begin{aligned}
g_{r}(y) & =g_{r}(x)\left(\operatorname{res}(x)\left(-\frac{1}{r t^{r}}\right)\right)^{-1} \sigma_{r}\left(\operatorname{res}(x)\left(-\frac{1}{r t^{r}}\right)\right) \\
& =g_{r}(x) \operatorname{res}(x)(-u t+\epsilon)
\end{aligned}
$$

by reporting the above identity (4.1). We have $g_{r}(y) \in G\left(A^{u}[[t]]\right)$ so that $r(y) \leq r$. The reduction mod $t$ of $g_{r}(y)$ is trivial by construction so that Proposition 4.1.(2) shows that $r(y)<r$, which contradicts the minimality of $r$.

Multiplicative case: $r=0$. Similarly we use the homomorphism $\operatorname{res}(x): \mathbb{G}_{m, A} \rightarrow G$ for constructing the point $y=x \cdot \operatorname{res}(x)\left(t^{-1}\right)^{-1}$. Since $G$ is commutative, we have

$$
\begin{aligned}
g_{0}(y) & =g_{0}(x) \operatorname{res}(x)\left(t^{-1}\right) \sigma_{0}\left(\operatorname{res}(x)\left(t^{-1}\right)\right)^{-1} \\
& =g_{0}(x) \operatorname{res}(x)(\lambda)^{-1} .
\end{aligned}
$$

We have $g_{0}(y) \in G\left(A^{+}[[t]]\right)$ so that $r(y) \leq 0$. The reduction $\bmod t$ of $g_{r}(y)$ is $\operatorname{res}(x)(\lambda) \operatorname{res}(x)\left(\lambda^{-1}\right)=1$ so that Proposition 4.1.(2) shows that $r(y)<0$. Thus $y \in X(A[[t]])$ and $X(A)$ is not empty.

\section{The CASE OF FIELDS, II}

Let $k$ be a field. A $k$-variety is a separated $k$-scheme of finite type. If $X$ is a $k$-variety, we denote by $X^{\dagger}$ its largest geometrically reduced $k$-subscheme [7, C.4.1].

A $k$-compactification of a $k$-variety $V$ is an open immersion $j: V \rightarrow V^{c}$ where $V^{c}$ is a proper $k$-variety.

We use intensively the notions of the book [7], for example the different radicals of a smooth algebraic $k$-group $H$. 
$\mathcal{R}_{u, k}(H): k$-unipotent radicial, i.e. the largest smooth connected unipotent normal $k$-subgroup of $H$.

$\mathcal{R}_{k}(H): \quad k$-radical, i.e. i.e. the largest smooth connected solvable normal $k-$ subgroup of $H$.

$\mathcal{R}_{u s, k}(H)$ : split unipotent $k$-radical, i.e. the largest smooth connected $k$-split unipotent normal $k$-subgroup of $H$.

$\mathcal{R}_{s, k}(H)$ : split $k$-radical i.e. the largest smooth connected $k$-split solvable normal $k$-subgroup of $H$.

5.1. Pseudo-complete varieties. We say that a $k$-variety is pseudo-complete if $X(A)=X(K)$ for each discretly valued $k$-ring $A$ with fraction fields $K$ and whose residue field is separable over $k$ [7, app. C.1.]. To check this property, it is enough to consider the case of a complete discretly valued $k$-ring whose residue field is separably closed and separable over $k$ (ibid, C.1.2.). In particular, $X$ is pseudo-complete if and only if $X_{k_{s}}$ is pseudo-complete.

We say that $X$ is $k$-pseudo-complete if $X(A)=X(F)$ for each discretly valued $F$ ring $A$ of fraction field $F$ and of residue field $k$. Similarly, it is enough to consider the case of $O=k[[t]]$ whose fraction field is denoted by $K=k((t))$. In particular an affine algebraic $k$-group $H$ is $k$-pseudo-complete if and only if $H$ is $k$-wound according to Corollary 3.8.

Lemma 5.1. Let $H$ be a $k$-algebraic group. We assume that $H$ is $k$-pseudo-complete. Let $f: Y \rightarrow X$ be a $H$-torsor where $Y, X$ are $k$-varieties. If $X$ is $k$-pseudo-complete, then $Y$ is $k$-pseudo-complete.

Proof. We are given $y \in Y(K)$ where $K=k((t))$. There exists a point $x \in X(O)$ such that $\pi(y)=x_{K}$. Let $\mathfrak{Z}=\pi^{-1}(x)$, this is a $H \times_{k} O$-torsor and $y \in \mathfrak{Z}(K)$. Since the $O$-group scheme $H \times{ }_{k} O$ is $O$-wound, Corollary 4.2 shows that the $O$-torsor $\mathfrak{Z}$ is trivial, that is there is a $H \times_{k} O$-equivariant isomorphism $\phi: \mathfrak{Z} \stackrel{\sim}{\longrightarrow} H \times_{k} O$. Since $H$ is $k$-wound, we have $H(O)=H(K)$ hence $\mathfrak{Y}(O)=\mathfrak{Y}(K)$. We conclude that $y \in Y(O)$.

5.2. Affine $k$-groups. We recall the notations $K=k((t))$ and $O=k[[t]]$.

Proposition 5.2. Let $G$ be an affine algebraic $k$-group. Let $P$ be a minimal pseudoparabolic $k$-subgroup of $\left(G^{\dagger}\right)^{0}$. We put $R=\mathcal{R}_{s, k}(G)$.

(1) The quotient $Q=P / R$ is $k$-wound.

(2) For each $G$-torsor $E$ over $k$ trivialized over $k_{s}, E / R$ is $k$-pseudo-complete and $E(k) \neq \emptyset$ if and only if $(E / R)(K) \neq \emptyset$.

(3) Let $E$ be a G-torsor. If $E(K) \neq \emptyset$, then $E(k) \neq \emptyset$.

Remarks 5.3. (a) In the perfect field case, statement (1) follows of a compactification result due to Borel-Tits [1, th. 8.2].

(b) Statement (3) was known for groups of multiplicative type [6, th. 4.1]. 
Proof. (1) We have $\mathcal{R}_{s, k}(P) \subseteq \mathcal{R}_{k}(P)$ and $\mathcal{R}_{k}(P) / \mathcal{R}_{s, k}(P)$ is a smooth connected solvable $k$-group $[8$, th. 5.4$]$ which fits in the exact sequence

$$
1 \rightarrow \mathcal{R}_{k}(P) / \mathcal{R}_{s, k}(P) \rightarrow P / \mathcal{R}_{s, k}(P) \rightarrow P / \mathcal{R}_{k}(P) \rightarrow 1 .
$$

Let us show first that $P / \mathcal{R}_{s, k}(P)$ does not contain any proper central split $k$-subtorus. Let $S \subset P / \mathcal{R}_{s, k}(P)$ be a central split $k$-subtorus; its inverse image $M$ in $P$ is normal and is an extension of $S$ by $\mathcal{R}_{s, k}(P)$. Then $M$ is solvable $k$-split and by maximality of $\mathcal{R}_{s, k}(P)$, it follows that $S=1$.

If $P / \mathcal{R}_{s, k}(P)$ admits a $k$-subgroup $U$ isomorphic to $\mathbb{G}_{a}, U$ cannot be a $k$-subgroup of the wound $k$-group $\mathcal{R}_{k}(P) / \mathcal{R}_{s, k}(P)$. It follows that the morphism $U \rightarrow P / \mathcal{R}_{k}(P)$ is non trivial. Since any non-trivial quotient of $\mathbb{G}_{a}$ is isomorphic to $\mathbb{G}_{a}$ [10, IV.2.1.1], it follows that $P / \mathcal{R}_{k}(P)$ admits a $k$-subgroup $V$ isomorphic to $\mathbb{G}_{a}$. We use now the fact that the quotient $P / \mathcal{R}_{k}(P)$ is pseudo-reductive. This implies that $V \subseteq \mathcal{R}_{u s, k}\left(Q^{\prime}\right)$ where $Q^{\prime}$ is a pseudo-parabolic $k$-subgroup of $P / \mathcal{R}_{k}(P)$ [7, C.3.8]. The inverse image $P^{\prime}$ of $Q^{\prime}$ in $P$ is a (proper) pseudo-parabolic $k$-subgroup of $P$ (ibid, 2.2.10), and in the same time a pseudo-parabolic $k$-subgroup of $\left(G^{\dagger}\right)^{0}(i b i d, 3.5 .5)$. This leads to a contradiction with the minimality of $P$. Thus $Q=P / \mathcal{R}_{s, k}(P)$ is $k$-wound.

(2) The smooth case. Let $E$ be a $G$-torsor over $k$ and consider the quotient $Y=E / R$. We claim that $X=E / P$ is pseudo-complete (and a fortiori $k$-pseudo-complete). Since $E\left(k_{s}\right) \neq \emptyset$, we have $(E / P)_{k_{s}} \cong(G / P)_{k_{s}}$. We recall that $(G / P)_{k_{s}}$ is pseudocomplete [7, C.1.6], so that $(E / P)_{k_{s}}$ is pseudo-complete. It follows that $X=E / P$ is pseudo-complete. We consider the morphism $f: Y \rightarrow X=E / P$ which is a $Q=P / R$-torsor. Since $Q$ is $k$-pseudo-complete, Lemma 5.1 enables us to conclude that $Y$ is $k$-pseudo-complete.

General case. Since $E\left(k_{s}\right) \neq \emptyset, E^{\dagger}$ is a $G^{\dagger}$-torsor which satisfies $E^{\dagger} \wedge^{G^{\dagger}} G=E$. We consider the morphism $G / R \rightarrow G / G^{\dagger}$ and its variant $q: E / R \rightarrow E / G^{\dagger}$. We have $\left(E / G^{\dagger}\right)(k)=\left(E / G^{\dagger}\right)(O)=\left(E / G^{\dagger}\right)(K)=\{\bullet\}$. The pre-image of this point is the $k$-scheme $E^{\dagger} / R$ (actually $\left.X^{\dagger}\right)$. It follows that $\left(E^{\dagger} / R\right)(K)=(E / R)(K)$. Appealing to the smooth case, we get $\left(E^{\dagger} / R\right)(O)=\left(E^{\dagger} / R\right)(K)$. Thus $(E / R)(O)=(E / R)(K)$.

For the second property, we assume that $X(k) \neq \emptyset$. The morphism $E \rightarrow E / R=X$ is a $R$-torsor with $R$ solvable and $k$-split. Since $H^{1}(k, R)=1$ (by dévissage from the cases of $\mathbb{G}_{m}$ and $\left.\mathbb{G}_{a}\right)$, we conclude that $E(k) \neq \emptyset$.

(3) Let $E$ be a $G$-torsor such that $E(K) \neq \emptyset$. Since $K$ is separable over $k$, we have $E^{\dagger}(K)=E(K)$ whence $E^{\dagger} \neq \emptyset$. But $E^{\dagger}$ is geometrically reduced and is then generically smooth $[23,32.25 .7]$. It follows that $E^{\dagger}\left(k_{s}\right) \neq \emptyset$ (ibid, 32.25.6). A fortiori we have $E\left(k_{s}\right) \neq \emptyset$ and the statement becomes a straightforward consequence of $(2)$.

5.3. Torsors over fields. Let $G$ be a $k$-algebraic group. We continue with the notations $K=k((t))$ and $O=k[[t]]$. Our purpose is to discuss the kernels of mappings $a_{G}: H^{1}(O, G) \rightarrow H^{1}(K, G), b_{G}: H^{1}(k, G) \rightarrow H^{1}(K, G), c_{G}: H^{1}(O, G) \rightarrow H^{1}(k, G)$. 
If $G$ is smooth, the specialisation $c_{G}: H^{1}(O, G) \rightarrow H^{1}(k, G)$ is bijective according to Hensel's lemma [22, XXIV.8.1].

Theorem 5.4. The map $b_{G}: H^{1}(k, G) \rightarrow H^{1}(K, G)$ is injective.

Lemma 5.5. Let $1 \rightarrow G_{1} \rightarrow G_{2} \rightarrow G_{3} \rightarrow 1$ be an exact sequence of $k$-algebraic groups such that $G_{3}(O)=G_{3}(K)$.

(1) We assume that $G_{3}$ is smooth. If $\operatorname{ker}\left(a_{G_{1}}\right) \subseteq \operatorname{ker}\left(c_{G_{1}}\right)$ and $\operatorname{ker}\left(a_{G_{3}}\right) \subseteq \operatorname{ker}\left(c_{G_{3}}\right)$, then $\operatorname{ker}\left(a_{G_{2}}\right) \subseteq \operatorname{ker}\left(c_{G_{2}}\right)$.

(2) If $\operatorname{ker}\left(b_{G_{1}}\right)=1$ and $\operatorname{ker}\left(b_{G_{3}}\right)=1$, then $\operatorname{ker}\left(b_{G_{2}}\right)=1$.

Proof. (1) It is a classical "dévissage", see for example the proof of [5, Th. 2.1]. The above sequence gives rise to the exact commutative diagram of pointed sets

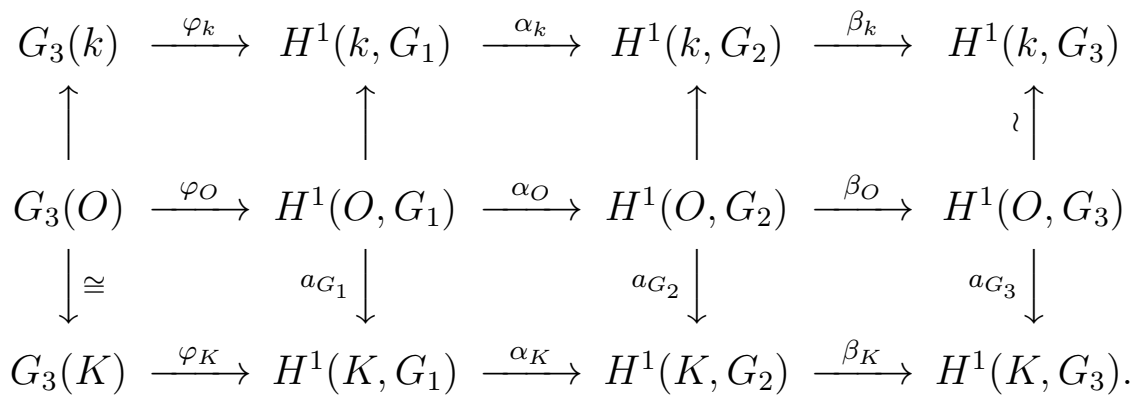

We are given an element $\gamma_{2} \in \operatorname{ker}\left(H^{1}\left(O, G_{2}\right) \rightarrow H^{1}\left(K, G_{2}\right)\right)$. Since $G_{3}$ is smooth, $a_{G_{3}}$ has trivial kernel and hence there exists a class $\gamma_{1} \in H^{1}\left(O, G_{1}\right)$ which maps on $\gamma_{2}$ and such that $\gamma_{1, K}$ belongs to the image of the characteristic map $\varphi_{K}$.

We use now the right action of $G_{3}(O)$ on $H^{1}\left(O, G_{1}\right)$ and the fibers of $\alpha_{O}$ are the orbits for that action [16, III.3.3.3]. The same fact holds for $G_{3}(K)$ and $\alpha_{K}$. It follows that there exists $g \in G_{3}(K)$ such that $\gamma_{1, K} \cdot g_{3}=1 \in H^{1}\left(K, G_{1}\right)$. On the other hand, we have $g_{3} \in G_{3}(O)$ hence $\gamma_{1, O} \cdot g_{3} \in \operatorname{ker}\left(H^{1}\left(O, G_{1}\right) \rightarrow H^{1}\left(K, G_{1}\right)\right)$. We can assume that $\gamma_{1} \in \operatorname{ker}\left(a_{G_{1}}\right)$. One of our assumption is that $\operatorname{ker}\left(a_{G_{1}}\right) \subseteq \operatorname{ker}\left(c_{G_{1}}\right)$ Thus $c_{G_{2}}\left(\gamma_{2}\right)=c_{G_{2}}\left(\alpha\left(\gamma_{1}\right)\right)=\alpha_{k}\left(c_{G_{1}}\left(\gamma_{1}\right)\right)=1 \in H^{1}\left(k, G_{2}\right)$.

The second fact is of the same vein.

We can proceed to the proof of Theorem 5.4.

Proof. In the affine case, this is Proposition 5.2.(3). We assume $G$ connected. According to Chevalley $\left[22, \mathrm{VI}_{B}\right.$.12.5.(5)], there is an exact sequence $1 \rightarrow H \rightarrow G \rightarrow$ $G / H \rightarrow 1$ where $G / H$ is an abelian variety and $H$ is affine. By application of the valuative criterion of properness to $A$-torsors, we have that $\operatorname{ker}\left(b_{A}\right)=1$. On the other hand, we have $\operatorname{ker}\left(b_{H}\right)=1$, so that Lemma 5.5.(2) shows that $\operatorname{ker}\left(b_{G}\right)=1$. To reach the non-connected case is of the same vein by using the exact sequence $1 \rightarrow G \rightarrow G \rightarrow G / G^{0} \rightarrow 1$. 


\section{Link With GABBER's COMPACTIFICATIONS}

6.1. The statements. The following two results have been announced by Gabber [20] and will not be used in the paper. Let $k$ be a field. If $G$ is an algebraic $k$-group, we denote by $L^{\prime} G$ its largest smooth affine connected $k$-subgroup, by $L_{\bar{k}} G$ the largest largest smooth affine connected subgroup of $G_{\bar{k}}$ and by $\bar{L} G$ the smallest $k$-subgroup such that $\bar{L} G \supset L_{\bar{k}} G$. We consider the following property (due to Gabber)

$$
\text { All tori of } G_{\bar{k}} \text { are in }\left(G^{\dagger}\right)_{\bar{k}} \text {. }
$$

Theorem 6.1. Let $G$ be a k-group of finite type and $P$ a pseudo-parabolic subgroup of $L^{\prime} G$. Then $G / P$ has an equivariant projective compactification, compatible with $\left(G^{\dagger}\right)^{0}, L^{\prime} G, \bar{L} G$, with a $G$-linearized line bundle relatively ample for $G / P \rightarrow G / \bar{L} G$ such that the boundary has no separable point and if $(*)$ holds there is no $k_{s}$-orbit contained set-theoretically in the boundary.

Theorem 6.2. Let $G$ be a k-group of finite type. Then $G$ admits a projective compactification $G \hookrightarrow G^{c}$ with a left action of $G$, right action of $G^{\dagger}$, an invariant ample effective divisor with support $G^{c} \backslash G$ if $G$ is affine, such that

(1) For every separable extension $K$ of $k$ the following are equivalent:

(i) $G_{K}$ has a subgroup isomorphic to $\mathbb{G}_{a}$ or $\mathbb{G}_{m}$;

(ii) $G(K) \neq G^{c}(K)$;

(iii) $\overline{L^{\prime} G}(K) \neq L^{\prime} G(K)$;

(iv) There is an $K$-orbit for the left action of $G$ on $G^{c}$ admitting a separable point and contained in $G^{c} \backslash G$.

(2) For every separably closed separable extension $K$ of $k, \bar{G}(K)=G^{\dagger}(K)=G(K) \overline{\left(L^{\prime} G\right)}(K)$, and if $(*)$ holds every $K$-orbit of the action of $G$ on $G^{c}$ has a $K$-point.

\subsection{Refinement of Theorem 5.4.}

Theorem 6.3. Let $G$ be an affine algebraic $k$-group which satisfies the property $(*)$. Then $\operatorname{ker}\left(a_{G}\right)=1$.

Proof. Once again we use the notations $O=k[[t]]$ and $K=k((t))$. We consider the exact sequence of fppf $k$-sheaves

$$
1 \rightarrow G^{\dagger} \rightarrow G \rightarrow G / G^{\dagger} \rightarrow 1
$$

We put $Q=G / G^{\dagger}, \mathfrak{G}=G \times_{k} O, \mathfrak{Q}=Q \times_{k} O$. According to Gabber [14, th. 5.2], $Q$ admits a $G$-equivariant compactification $Q^{c}$ such that the boundary $\partial Q=Q^{c} \backslash Q$ has no $G$-orbit over any separable field extension of $k$. Furthermore $Q^{c}$ carries a $G$-linearized line bundle. We put $\mathfrak{Q}^{c}=Q^{c} \times_{k} O$. Let $\mathfrak{X}$ be a $\mathfrak{G}$-torsor such that $\mathfrak{X}_{K}$ is trivial, that is $\mathfrak{X}(K) \neq \emptyset$. We consider the contracted products $\mathfrak{Z}=\mathfrak{X} \wedge^{G} \mathfrak{Q}$ and $\mathfrak{Z}^{c}=\mathfrak{X} \wedge^{\mathfrak{G}} \mathfrak{Q}^{c}$ (which are representable according to Lemma 10.1) over $O$. We put $X=\mathfrak{X}_{k}, Z=\mathfrak{Z}_{k}$ and $Z^{c}=\mathfrak{Z}_{k}^{c}$.

Claim 6.4. $Z(k)=Z^{c}(k)$. 
The argument is similar with that of the proof of $[14$, Lemme 6.1]. We consider the $k$-group scheme $G^{\prime}=\operatorname{Aut}_{G}(X)$, it acts (on the left) on the morphism $X \rightarrow Z$. Let $z \in Z^{c}(k)$. According to [14, 2.3.2], the $k$-orbit $T$ of $Z$ under $G^{\prime}$ corresponds canonically to a $k$-orbit $T_{0}$ of $G$ on $Q^{c}$. By assumption, we have $T_{0} \subset Q$, so that $T \subset Z$. In particular $z \in Z(k)$ and the Claim is proven.

We have compatible isomorphisms $\mathfrak{Z}_{K}^{c} \stackrel{\sim}{\longrightarrow} \mathfrak{Q}^{c} \times_{k} K$ and $\mathfrak{Z}_{K} \stackrel{\sim}{\longrightarrow} \mathfrak{Q} \times{ }_{k} K$. Since $\{\bullet\}=\mathfrak{Q}(K)=\mathfrak{Q}^{c}(K)$, we have that $\left\{z_{0}\right\}=\mathfrak{Z}(K)=\mathfrak{Z}^{c}(K)$. The Claim implies that the specialization of $z_{0}$ in $Z^{c}(k)$ belongs to $Z(k)$. It follows that $z_{0} \in \mathfrak{Z}(O)$ so that $\left\{z_{0}\right\}=\mathfrak{Z}(O)$. Hence the $\mathfrak{G}$-torsor $\mathfrak{X}$ admits a reduction $\mathfrak{F}$ to the subgroup $G^{\dagger} \times_{k} O$. The exact commutative diagram

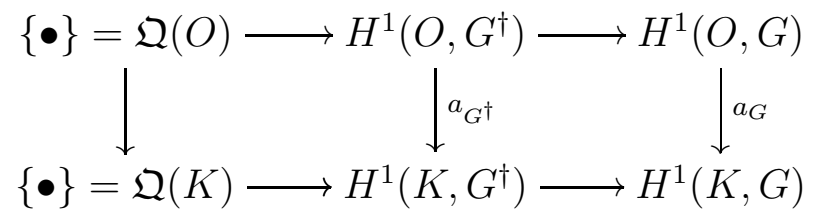

enables us to conclude that $\mathfrak{X}$ is a trivial $\mathfrak{G}$-torsor.

\section{Applichtions AND EXAmples}

7.1. Applications. We shall apply the main result to nice torsors.

Corollary 7.1. Let $G$ be an algebraic $k$-group.

(1) We assume that $G$ acts (on the left) on a $k$-variety $X$. Let $x, x^{\prime} \in X(k)$. Then $x$ and $x^{\prime}$ are $G(k)$-conjugated if and only if $x_{K}$ and $x_{K}^{\prime}$ are $G(K)$-conjugated.

(2) Let $H, H^{\prime}$ be $k$-subgroups of $G$. Then $H$ and $H^{\prime}$ are $G(k)$-conjugated if and only if $H_{K}$ and $H_{K}^{\prime}$ are $G(K)$-conjugated.

Proof. (1) We assume that $x, x^{\prime}$ are $G(K)$-conjugated. We consider the transporter $E=\left\{g \in G \mid g \cdot x=x^{\prime}\right\}$. Since $E(K) \neq \emptyset, E$ is non-empty and is a torsor under the stabilizer $G_{x}=\{g \in G g \cdot x=x\}$. Theorem 5.4 yields that $E(k) \neq \emptyset$. Thus $x$ and $x^{\prime}$ are $G(k)$-conjugated.

(2) We assume that $H$ and $H^{\prime}$ are $G(K)$-conjugated. We denote by $N=N_{G}(H)$ the normalizer of $H$ in $G$. We consider the strict transporter $T$ of $H$ to $H^{\prime}$ as defined in $\left[10, \mathrm{VI}_{B} \cdot 6.2 .4\right]$. Since $T(K)$ is non-empty, $T$ is a $N$-torsor. Theorem 5.4 yields that $T(k) \neq \emptyset$. Thus $H$ and $H^{\prime}$ are $G(k)$-conjugated.

Another useful statement is the following.

Corollary 7.2. Let $X$ be a $k$-variety (resp. an algebraic $k$-group, etc..) whose automorphism group is representable by an algebraic $k$-group. Let $X^{\prime}$ be a $k$-form of $X$. Then $X$ and $X^{\prime}$ are $k$-isomorphic if and only if $X_{K}$ and $X_{K}^{\prime}$ are $K$-isomorphic.

Proof. We apply Theorem 3.4 to the $\operatorname{Aut}(X)$-torsor $\operatorname{Isom}\left(X, X^{\prime}\right)$. 
7.2. Examples of $k$-groups such that $\operatorname{ker}\left(a_{G}\right) \neq 1$. We assume that $k$ is imperfect of characteristic $p>0$ and we pick an element $a \in k \backslash k^{p}$.

(a) Let $\alpha_{p}=\operatorname{Spec}\left(k[t] / t^{p}\right)$, this is a finite closed $k$-subgroup of $\mathbb{G}_{a}$. We denote by $G_{0}=\operatorname{Aut}\left(\alpha_{p}\right)$ the $k$-group (affine, algebraic) of automorphisms of the finite $k$-scheme $\alpha_{p}$. The pointed set $H^{1}\left(k, G_{0}\right)$ classifies the $k$-forms of the $k$-algebra $A_{0}=k[x] / x^{p}$ [10, III.5.1.10]. The $k$-algebra $A=k[x] /\left(x^{p}-a\right)$ is a $k$-form of $A_{0}$ and we denote by $G$ its automorphism group; $G$ is a $k$-form of $G_{0}$.

We consider now the $O$-algebra $B=O[x] /\left(x^{p}-a t^{p}\right)$, which is an $O$-form of $A \times{ }_{k} O$. This gives rise to a class $[B] \in H^{1}(O, G)$. Since $A \otimes_{k} K \stackrel{\sim}{\longrightarrow} B \otimes_{O} K,[B]$ has trivial image in $H^{1}(K, G)$. On the other hand, $a$ is not a $p$-power in $B$ so that $A \otimes_{k} O$ and $B$ are not $O$-isomorphic. Thus $[B] \neq 1 \in H^{1}(O, G)$ and $\operatorname{ker}\left(a_{G}\right) \neq 1$.

(b) We shall construct an example of dimension 1 which arises from [14, §7.1]. We consider the action of the $k$-group $G:=\mathbb{G}_{a} \rtimes \mathbb{G}_{m}$ (semi-direct product for the standard action of $\mathbb{G}_{m}$ on $\mathbb{G}_{a}$ ) on the affine line $\mathbf{A}_{k}^{1}$ defined by

$$
(x, y) . z=x^{p}+y^{p} z \quad\left(x \in \mathbb{G}_{a}, y \in \mathbb{G}_{m}, z \in \mathbf{A}^{1}\right) .
$$

We observe that $\mathbf{A}_{k}^{1}$ is a homogeneous $K$-space (on the left) under $G$; also the stabilizer of 0 is the closed $k$-subgroup $\alpha_{p} \rtimes_{k} \mathbb{G}_{m}$. For an element $t_{0} \in O$, we denote by $\mathfrak{G}_{t_{0}}$ the stabilizer of $t_{0}$, i.e.

$$
\mathfrak{G}_{t_{0}}=\left\{(x, y) \in G \times_{k} O \mid x^{p}+y^{p} t_{0}=t_{0}\right\} .
$$

We are given $t_{1} \in O$, the strict transporter from $t_{0}$ to $t_{1}$ is the $\left(\mathfrak{G}_{t_{0}}, \mathfrak{G}_{t_{1}}\right)$-bitorsor

$$
\mathfrak{E}_{t_{0}, t_{1}}=\left\{(x, y) \in G \times_{k} O \mid x^{p}+y^{p} t_{0}=t_{1}\right\} .
$$

We consider the special cases $t_{0}=a$ and $t_{1}=a t^{p}$ which is taylor made for having $\mathfrak{E}_{t_{0}, t_{1}}(K) \neq \emptyset$. Since $a \notin k^{p}$, one has $\mathfrak{E}_{t_{0}, t_{1}}(k)=\emptyset$ and a fortiori $\mathfrak{E}_{t_{0}, t_{1}}(O)=\emptyset$.

The point is that the $O$-scheme $\mathfrak{G}_{a}$ arises from the $k$-scheme $H=\{(x, y) \in G \mid$ $\left.x^{p}+y^{p} a=a\right\}$. We conclude that the map $a_{H}: H^{1}(O, H) \rightarrow H^{1}(K, H)$ has a non trivial kernel. Furthermore we have $\operatorname{ker}\left(a_{H}\right) \not \subset \operatorname{ker}\left(c_{H}\right)$.

Remark 7.3. Theorem 6.3 shows that the $k$-group $G$ of Example (a) (resp. $H$ of Example (b)) does not satisfy property $(*)$. This fact can be checked directly.

(a) The $k$-group $G_{0}$ contains $\mathbb{G}_{m}$ so that $G_{\bar{k}}$ contains $\mathbb{G}_{m, \bar{k}}$. On the other hand, we have $G\left(k_{s}\right)=\operatorname{Aut}\left(k_{s}[x] /\left(x^{p}-a\right)=1\right.$ so that $G^{\dagger}=1$.

(b) We have $H\left(k_{s}\right)=1$ so that $H^{\dagger}=1$. On the other hand, we have $H_{\bar{k}} \cong\left(\alpha_{p} \rtimes \mathbb{G}_{m}\right)_{\bar{k}}$ which contains $\mathbb{G}_{m, \bar{k}}$.

\section{A MORE ADVANCED VIEWPOINT}

In this section, our goal is to give an abstract exposition of the group structure on automorphisms of $A[[t]]$ used in the second section. 


\subsection{Automorphisms of Laurent series and pro-group schemes. .}

For each $w \geq 0$, we consider the affine $\mathbb{Z}$-group scheme $J_{w}$ of automorphisms of the sequence of rings

$$
\mathbb{Z}[t] / t^{w+1} \rightarrow \mathbb{Z}[t] / t^{w} \rightarrow \cdots \rightarrow \mathbb{Z}
$$

For each ring $Z, J_{w}(R)$ consists of elements $f_{i} \in \operatorname{Aut}_{R-\text { ring }}\left(R[t] / t^{i}\right)$ for $i=0, \ldots, w+1$ such that $f_{i}=f_{i+1} \bmod t^{i}$ for $i=0, \ldots, w$. We have transition maps $\pi^{w, w-1}: J_{w} \rightarrow$ $J_{w-1}$ for each $w \geq 1$. The projective limit in the sense of $[12, \S 8]$ is denoted by $J=$ $\lim J_{w}$. This is an affine $\mathbb{Z}$-group scheme whose coordinate ring is $\mathbb{Z}[J]=\underline{\lim } \mathbb{Z}\left[J_{w}\right]$. We have projections $j_{w}: J \rightarrow J_{w}$ and we put $J^{w}=\operatorname{ker}\left(j_{w}\right)$. Since $J_{0}$ is trivial, we have $J^{0}=J$. The following statement is straightforward.

Lemma 8.1. Let $w \geq 1$ and let $A$ be a ring.

(1) An element of $J_{w}(A)$ is given by $t \mapsto a_{1} t+a_{2} t^{2}+\cdots+a_{w} t^{w}$ with $a_{1} \in A^{\times}$and $a_{2}, \ldots, a_{n} \in A$.

(2) We have an exact sequence $1 \rightarrow \mathbb{G}_{a} \rightarrow J_{w+1} \rightarrow J_{w} \rightarrow 1$.

In particular, we have $J_{1}=\mathbb{G}_{m}$ and the map $j^{1}: J \rightarrow J_{1}=\mathbb{G}_{m}$ is split by mapping a scalar $\lambda \in R^{\times}$to $f_{i}=\times \lambda$. It follows that $J=J^{1} \rtimes \mathbb{G}_{m}$ where $J^{1}$ is a pro-unipotent $\mathbb{Z}$-group scheme.

For a ring $R$, we have $R[[t]]=\varliminf^{\lim } R[t] / t^{w+1}$ so that $J(R)=\underline{\lim } J_{w}(R)$ acts on $R[[t]]$. We name $J(R)$ the group of continuous automorphisms of $R[[t]]$. An important thing is that $J(R)$ acts also on $A[[t]]$ for each $R$-algebra $A$.

8.2. Torsors and cocycles for Hochschild cohomology. We are given a ring $A$, an $A$-group scheme $G$ equipped with a closed immersion $G \hookrightarrow \mathrm{SL}_{N}$, a $G$-torsor $X$ and a point $x \in X(A((t)))$. Of course an important case is $G$ itself. For each $A$-ring $B$ and each $\sigma \in J(B)$ we write $\sigma\left(x_{B}\right)=x_{B} \cdot z_{\sigma}(x)$ with $z_{\sigma}(x) \in G(B((t)))$.

For each $w \geq 0$ we denote by $G_{w}=\prod_{A[t] / t^{w+1} /{ }_{A}} G_{A[t] / t^{w+1}}$ the Weil restriction of $G$ with respect to the finite $A$-algebra $A[t] / t^{w(x)+1}$.

We denote by $L^{+} G$ the $A$-functor in groups $B \mapsto G(B[[t]])$; there is a natural map $L^{+} G \rightarrow G_{w}$ for each $w \geq 0$. Similarly we denote by $L G$ the $A$-functor in groups $B \mapsto G(B((t)))$.

Lemma 8.2. There exists a smallest integer $w(x)$ such that the restriction of $z(x)$ to $J^{w(x)}$ factorizes through $L^{+} G$.

Proof. We have to prove that there exists $w \in \mathbb{N}$ such that $z_{\sigma}(x) \in G(B[[t]])$ for all $R$-algebras $B$ and all $\sigma \in J(B)$. Without loss of generality, we can replace $A$ by a faithfully flat extension so we can assume that $X$ is a trivial $G$-torsor and $x=g \in G(A((t)))$. We can replace then $G$ by $\mathrm{SL}_{N}$ and use the setting of the proof of Proposition 2.1. We write $g=t^{-d} \underline{g}$ with $d \geq 0$ and $\underline{g} \in \mathrm{M}_{N}(A[[t]]) \backslash t \mathrm{M}_{N}(A[[t]])$. 
It follows that $\operatorname{det}(\underline{g})=t^{N d}$. For an $A$-algebra $B$ and $\sigma \in J(B)$, we have

$$
g_{B[[t]]}^{-1} \sigma\left(g_{B[[t]]}\right)=\left(\frac{\sigma(t)}{t}\right)^{d} \underline{g}_{B[[t]]}^{-1} \sigma\left(\underline{g}_{B[[t]]}\right) .
$$

We write $\underline{g}=\left(P_{i, j}\right)_{i, j=1, . ., N}$ with $P_{i, j} \in A[[t]]$ and denote by $\Delta_{i, j} \in A[[t]]$ the minor of index $(i, j)$ of $\underline{g}$. We have $\underline{g}^{-1}=\left(t^{-N d} \Delta_{i, j}\right)_{i, j=1, . ., N}$ so that the $(i, j)$-coefficient $D_{i, j, \sigma}$ of $g_{B[[t]]}^{-1} \sigma\left(g_{B[[t]]}\right)$. is

$$
D_{i, j, \sigma}=\left(\frac{\sigma(t)}{t}\right)^{d} t^{-N d} \sum_{k=1}^{N} \Delta_{i, k}(t) P_{k, j}(\sigma(t)) \in B((t)) .
$$

When $\sigma=1, C_{i, j, \sigma}$ specializes on $\delta_{i, j}$ so that

$$
D_{i, j, \sigma}=\delta_{i, j}+\left(\frac{\sigma(t)}{t}\right)^{d} t^{-N d} \sum_{k=1}^{N} \Delta_{i, k}(t)\left(P_{k, j}(\sigma(t))-P_{k, j}(t)\right) .
$$

It follows that there exists a uniform integer $w \geq 0$ such that $g_{B[[t]]}^{-1} \sigma\left(g_{B[[t]]}\right)$ belongs to $G(B[[t]])$ for $\sigma \in J^{w}(B)$.

Our construction defines then a 1-cocycle $z(x): J^{w(x)} \rightarrow L^{+} G$ for the Hochschild cohomology as defined by Demarche $[9, \S 2.1]$ for the $A$-functor in groups $J^{w(x)}$ and $L G$. This induces an 1-cocycle for the Hochschild cohomology

$$
\operatorname{Res}(x): J^{w(x)} \rightarrow G_{w(x)} .
$$

Since $J^{w(x)}$ acts trivially on $G_{w(x)}$, the map $\operatorname{Res}(x)$ is actually a homomorphism of $A$-groups. This defines classes $\gamma(x)=[z(x)] \in H_{c o c}^{1}\left(J^{w(x)}, L G\right)$ and $[\operatorname{Res}(x)] \in$ $H_{c o c}^{1}\left(J^{w(x)}, G_{w(x)}\right)$.

Lemma 8.3. Let $A^{\prime}$ be a flat cover of $A$. Then $w\left(x_{\left.A^{\prime}(t)\right)}\right)=w(x)$ and $\operatorname{Res}\left(x_{\left.A^{\prime}((t))\right)}\right)=$ $\operatorname{Res}(x)$.

Proof. We have the obvious relation $w^{\prime}:=w\left(x_{\left.A^{\prime}(t)\right)}\right) \leq w(x)=: w$ (which holds in general). We observe that $w=0$ implies that $w^{\prime}=0$ as well. We can assume $w \geq 1$. By definition of $w$, there exists a ring extension $B$ of $A$ and $\sigma \in J^{w-1}(B)$ such that $z_{\sigma}(x) \in G(B((t))) \backslash G(B[[t]])$. We consider $B^{\prime}=A^{\prime} \otimes_{A} B$, this is a flat cover of $B$ so that $B$ injects in $B^{\prime}$. It follows that $B[[t]]=B^{\prime}[[t]] \cap B((t))$. Since $G$ is affine, we get that $\left(z_{\sigma}(x)\right)_{B^{\prime}}$ belongs to $G\left(B^{\prime}((t))\right) \backslash G\left(B^{\prime}[[t]]\right)$. Thus $w^{\prime}>w-1$ and $w^{\prime}=w$.

8.3. Compararison with the elementary construction. For each integer $r \geq 1$, we consider the map of $\mathbb{Z}$-functors ${ }^{2} \phi^{r}: \mathbb{G}_{a} \rightarrow J$, which associates to the coordinate $u$ the element of $J(\mathbb{Z}[u])$ defined by $t \mapsto t\left(1+u t^{r}\right)=t+u t^{r+1}$. We observe that $\phi$ factorizes through $J^{r+1}$.

\footnotetext{
${ }^{2}$ It is not a group functor.
} 
Lemma 8.4. The composite $\mathbb{G}_{a} \stackrel{\phi^{r}}{\rightarrow} J^{r} \rightarrow J^{r} / J^{r+1}$ is a $\mathbb{Z}$-group isomorphism.

Proof. We take two parameters $u_{1}, u_{2}$ and see that $\phi^{r}\left(u_{1}\right) \circ \phi^{r}\left(u_{2}\right) \in J\left(\mathbb{Z}\left[u_{1}, u_{2}\right]\right)$ maps $t$ to

$$
\left(t+u_{2} t^{r+1}\right)+u_{1}\left(t+u_{2} t^{r+1}\right)^{r+1}=t+\left(u_{1}+u_{2}\right) t^{r}+(r+1) u_{1} u_{2} t^{r+2}+\ldots
$$

The following statement is then obvious.

Lemma 8.5. For each integer $w \geq 1$ and each ring $B$, we have

$$
J^{w}(B)=\left\langle\phi^{r}(B)\right\rangle_{r \geq w} .
$$

We consider firstly the case when the index of $x$ is integral.

Lemma 8.6. We assume that $r(x) \in \mathbb{N}$.

(1) If $r(x) \geq 1$, then $w(x)=r(x)+1$ and the following diagrams
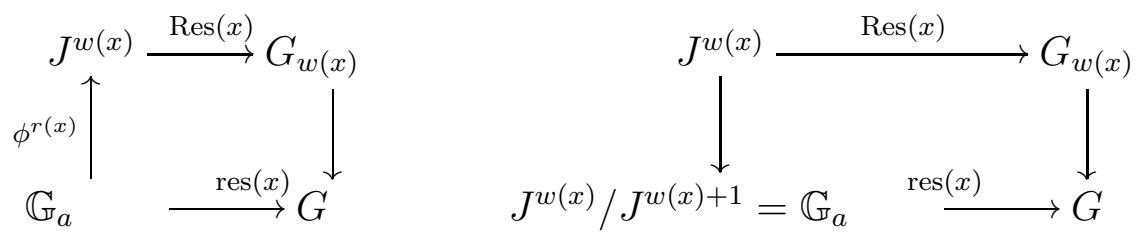

commute. Furthermore $\operatorname{Res}(x)$ is trivial on $J^{2 w(x)+1}$.

(2) If $r(x)=0$, then $w(x)=0$ or 1 and the following diagrams
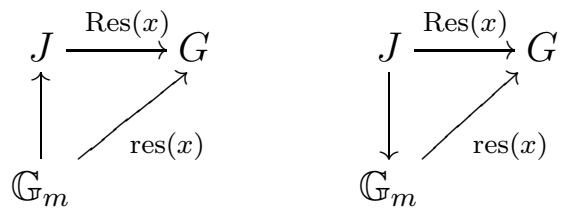

commute.

Proof. Once again we can localize for the flat topology and assume that the $G$-torsor is trivial.

(1) Since the maps $\phi^{r}$ factorize by $L^{+} G$ for all $r \geq r(x)$, it follows that $w(x) \leq$ $r(x)$ according to the identity of Lemma 8.5. On the other hand, $\phi^{r(x)-1}$ does not factorize by $L_{G}^{+}$, so that $w(x)>r(x)-1$. We conclude that $w(x)=r(x)$. The commutativity of the left-hand side diagram is clear. For the right hanside diagram we apply lemma 2.4.(1) to $r(g)$ and $M=1$. For $s \geq r(x)+1$, it follows that $g^{-1} \sigma_{s}(g) \in \operatorname{ker}\left(G\left(A^{u}[[t]]\right) \rightarrow G\left(A^{u}[t] / t\right)\right)$ so that

$$
\phi^{s}(A) \subseteq \operatorname{ker}\left(J^{w(x)}(A) \stackrel{\text { Res }}{\longrightarrow} G\left(A[t] / t^{w(x)+1}\right) \rightarrow G(A)\right)
$$


Lemma 8.5 yields that

$$
J^{w(x)+1}(A) \subseteq \operatorname{ker}\left(J^{w(x)}(A) \stackrel{\text { Res }}{\longrightarrow} G\left(A[t] / t^{w(x)+1}\right) \rightarrow G(A)\right) .
$$

Since we have the same property for each $A$-algebra $B$, the right-hand side compatibility is established. The last assertion follows from Lemma 2.4.(1) applied to $M=r(x)+1$.

(2) This is similar with (1) and the last assertion follows from Lemma 2.4.(2).

Remarks 8.3.1. (a) Since Res is a group homomorphism, Lemma 8.6 shows that res is a group homomorphism. We have then an alternative proof of the homomorphism part of Theorem 3.4.(1) and (2) when the index is integral. However we still need Proposition 2.1 for showing that $\operatorname{res}(x)$ (and a fortiori $\operatorname{Res}(x)$ ) are not trivial.

(b) In case (1), for $v=0, \ldots, w(x)$, the same argument shows more generally that Res : $J^{w(x)} \rightarrow G_{w(x)} \rightarrow G_{v}$ factorizes through $J^{w(x)} / J^{w(x)+v+1}$.

\section{LOOP GROUPS AND AFFINE GRASSMANNIANS}

Our goal is to relate our construction with the theory of affine grassmannians.

9.1. Ind-schemes. We continue with the same setting with $G \subset \mathrm{SL}_{N}=: H$ a closed $A$-subgroup scheme. The loop groups are the $A$-functors

$$
B \mapsto L_{G}^{+}(B)=G(B[[t]]), \quad B \mapsto L_{G}(B)=G(B((t))) .
$$

Both $A$-functors are equipped with the rotation action of $\mathbb{G}_{m}$ : for each $A$-ring $B$, each $b \in B^{\times}$and each $g \in L_{G}(B)=G(B((t)))$ we put $\delta(b) \cdot g=g\left(b^{-1} t\right)$. The $A$-functor $L_{G}^{+}$is representable by an $A$-scheme and the $A$-functor $L_{G}$ is representable by an ind $A$-scheme. More precisely $L_{H}$ is the union of the subfunctors $L_{H, d}$ given by those matrices $A$ for which the entries of $A$ are Laurent series of the form $\sum_{i>-d} a_{i} t^{i}$. Each $L_{H, d}$ is representable by an affine $A$-scheme and we set $L_{G, d}=L_{H, d} \cap \bar{L}_{G}$.

For $n \geq 1$, we denote by ${ }_{n} L_{G}(B)=G\left(B\left(\left(t^{1 / n}\right)\right)\right)$ and similarly for $L_{G}^{+}$. As in the introduction, we consider the polynomial ring $B^{u}=B[u]$ seen as a subring of the Laurent polynomial ring $B\left[\lambda, \lambda^{-1}\right]$ by applying $u$ to $\lambda-1$. We have a natural morphism $L_{G} \rightarrow{ }_{n} L_{G}$. Let $r=m / n \in \mathbb{Q}_{\geq 0}$ and consider the $A$-subfunctors of $L_{G}$ defined by

$$
{ }^{r} L_{G}(B)=\left\{[g] \in L_{G}(B) \mid g^{-1} \sigma_{r}(g) \in G\left(B^{u}\left[\left[t^{1 / n}\right]\right]\right)\right\}
$$

if $r>0$ and

$$
{ }^{0} L_{G}(B)=\left\{[g] \in L_{G}(B) \mid g^{-1} g(\lambda t) \in G\left(B\left[\lambda, \lambda^{-1}\right]\left[\left[t^{1 / n}\right]\right]\right)\right\} .
$$

For $r>s \geq 0$, we have ${ }^{s} L_{G} \subseteq{ }^{r} L_{G}$ according to Proposition 2.1 (resp. Lemma 2.6) in the case $s>0$ (resp. $s=0$ ). 
Lemma 9.1. (1) For each $d \in \mathbb{Z}_{\geq 0},{ }^{r} L_{G, d}=L_{G, d} \cap{ }^{r} L_{G}$ is a closed A-subscheme of $L_{G, d}$ and even of $L_{G, d / N}$.

(2) ${ }^{r} L_{G}$ is a closed ind-subscheme of $L_{G}$.

Proof. (1) Once again one can work with $H=\mathrm{SL}_{N}$. First case: $r>0$ : For each $d \geq 0$, we have by definition

$$
{ }^{r} L_{H, d}(B)=\left\{g \in L_{H, d}(B) \mid g^{-1} \sigma_{r}(g) \in \mathrm{SL}_{N}\left(B^{u}\left[\left[t^{1 / n}\right]\right]\right)={ }_{n} L_{H, d}\left(B^{u}\right)\right\} .
$$

We consider the $\mathbf{A}_{A}^{1}$-morphism $\psi_{r, d}: L_{H, d} \times{ }_{A} \mathbf{A}_{A}^{1} \rightarrow{ }_{n} L_{H, n N d} \times{ }_{A} \mathbf{A}_{A}^{1}$ defined by $(g, u) \mapsto g^{-1} \sigma_{r}(g)$. The $A$-functor ${ }^{r} L_{H, d}$ is the $A$-subfunctor of $L_{H, d}$ consisting in the $g \in L_{H, d}$ such that the map $u \mapsto g^{-1} \sigma_{r}(g)=\psi_{r, d}(g)$ factorizes through the closed $A$-subscheme ${ }_{n} L_{H, 0} \times{ }_{A} \mathbf{A}_{A}^{1}$. Since $\mathbb{G}_{a, A}$ is free over $A$ (i.e. $A[t]$ is a free $A$-module), it follows that the $A$-functor ${ }^{r} L_{H, d}$ is representable by a closed $A$-subscheme of $L_{H, d}$ according to $\left[22, \mathrm{VI}_{B} \cdot 6.2 .4\right]$. According to Corollary 3.5, for each $A$-field $E$, we have ${ }^{r} L_{H, d}(E) \subseteq L_{H, N / d}(E)$ so that ${ }^{r} L_{H} \subseteq L_{H, N / d}$.

Case $r=0$. The preceding argument works verbatim with the morphism $\psi_{d}: L_{H, d} \times_{A} \mathbb{G}_{m, A} \rightarrow L_{H, N d} \times_{A} \mathbb{G}_{m, A},(g, \lambda) \mapsto g^{-1} g(\lambda t)$.

(2) This is a straightforward consequence of (1).

Remark 9.2. The ${ }^{r} L_{G}$ 's have no reason to be $A$-schemes and this happens already for $\mathbb{G}_{m}$ since ${ }^{0} L_{\mathbb{G}_{m}}=L_{\mathbb{G}_{m}}$.

By specialization at $t=0$, we get the residue $A$-functors

$$
{ }^{r} \operatorname{res}_{G}:{ }^{r} L_{G} \rightarrow \operatorname{Hom}_{g r}\left(\mathbb{G}_{a}, G\right)(r>0),{ }^{0} \operatorname{res}_{G}:{ }^{0} L_{G} \rightarrow \operatorname{Hom}_{g r}\left(\mathbb{G}_{m}, G\right) .
$$

By definition, the affine grassmannian $\mathcal{Q}_{G}$ of $G$ is the fppf sheafification of the $A-$ functor

$$
B \mapsto \mathcal{F}(B)=G(B((t))) / G(B[[t]]) .
$$

Let $r \in \mathbb{Q}_{\geq 0}$ and consider the $A$-subfunctor of $\mathcal{F}$ defined by

$$
\mathcal{F}^{r}(B)=\left\{[g] \in{ }^{r} L_{G}(B) / L_{G}^{+}(B)\right\} .
$$

It is an $A$-subfunctor granting to Lemma 2.7.(2) and we denote its fppc sheafification by ${ }^{r} \mathcal{Q}_{G}$. Clearly the map $L G \rightarrow \mathcal{Q}_{G}$ induces an isomorphism $\left({ }^{r} L_{G} / L_{G}^{+}\right)_{f p p f} \stackrel{\sim}{\longrightarrow}{ }^{r} \mathcal{Q}_{G}$. Also the residue $A$-functors give rise to the $A$-functors

$$
{ }^{r} \operatorname{res}_{G}:{ }^{r} \mathcal{Q}_{G} \rightarrow \overline{\operatorname{Hom}_{g r}}\left(\mathbb{G}_{a}, G\right)(r>0),{ }^{0} \operatorname{res}_{G}:{ }^{0} \mathcal{Q}_{G} \rightarrow \overline{\operatorname{Hom}_{g r}}\left(\mathbb{G}_{m}, G\right)
$$

where $\overline{\operatorname{Hom}_{g r}}\left(\mathbb{G}_{a}, G\right)$ is the fppf quotient $\operatorname{Hom}_{g r}\left(\mathbb{G}_{a}, G\right) / G$ and similarly for $\mathbb{G}_{m}$.

Lemma 9.3. ${ }^{0} \mathcal{Q}_{G}$ is the fixed locus for the rotation action of $\mathbb{G}_{m}$ on $\mathcal{Q}_{G}$. 
Proof. The fixed locus ${ }^{\sharp} \mathcal{Q}_{G}$ is the $A$-subfunctor of $\mathcal{Q}_{G}$ defined by

$$
{ }^{\sharp} \mathcal{Q}_{G}(B)=\left\{x \in \mathcal{Q}_{G}(B) \mid x(c t)=x(t) \forall B \text {-algebra } C \text { and } \forall c \in C^{\times}\right\} .
$$

Clearly ${ }^{0} \mathcal{Q}_{G}$ is an $A$-subfunctor of ${ }^{\sharp} \mathcal{Q}_{G}$. Conversely we are given an $A$-algebra $B$ and an element $x \in{ }^{\sharp} \mathcal{Q}_{G}(B)$. To show that $x$ is fixed is local for the fppf topology so that we may assume that $x$ lifts to an element $g \in L_{G}(B)$. We take $C=B\left[\lambda^{ \pm 1}\right]$ so that $x(\lambda t)=x(t) \in \mathcal{Q}_{G}(C)$ It follows that $g(\lambda t)=g(t) h$ with $h \in L_{G}^{+}(C)$. hence $g^{-1} g(\lambda t) \in G\left(B\left[\lambda^{ \pm 1}\right][[t]]\right)$. In other words $g$ belongs to ${ }^{0} L_{G}(B)$ and $x \in{ }^{0} \mathcal{Q}_{G}(B)$.

9.2. Ind-schemes, II. Now we assume that the quotient $\mathrm{SL}_{N} / G$ is representable by an quasi-affine $A$-scheme. In this case, the structure of ind $A$-scheme of $\mathcal{Q}_{\mathrm{SL}_{N}}$ induces a structure of ind $A$-scheme on $\mathcal{Q}_{G}$ such that the map $\mathcal{Q}_{G} \rightarrow \mathcal{Q}_{\mathrm{SL}_{N}}$ is a locally closed immersion (which is closed if $\mathrm{SL}_{N} / G$ is affine), see [17, lemma 2.14].

Proposition 9.4. ${ }^{r} \mathcal{Q}_{G}$ is a closed $A$-ind-subscheme of $\mathcal{Q}_{G}$.

Proof. It is enough to consider the case of $\mathrm{SL}_{N}$. For each $d \geq 0$, the map $\mathrm{SL}_{N, d} \rightarrow \mathcal{Q}_{H, d}$ is a $L_{H}^{+}$-torsor (locally trivial for the étale topology). It follows that the quotient sheaf ${ }^{r} \mathrm{SL}_{N, d} / L_{H}^{+}$is representable by a closed $A$-subscheme of the $A$-scheme $\mathcal{Q}_{H, d}$. Thus ${ }^{r} \mathcal{Q}_{G}$ is a closed $A$-ind-subscheme of $\mathcal{Q}_{G}$.

9.3. Case of a split reductive $k$-group. We work here over a base field $k$ and assume that $G$ is a split reductive $k$-group equipped with a $\operatorname{Killing}$ couple $(B, T)$. We denote by $\Phi=\Phi(G, T)$ the associated root system and by $\Delta$ the subset of simple roots with respect to $B$. For each $\alpha \in \Phi$, we denote by $U_{\alpha}$ the image of the root homomorphism $u_{\alpha}: \mathbb{G}_{a} \rightarrow G$ attached to $\alpha$. We shall use also the $k$-group $L_{G}^{<0}$ defined by $L_{G}^{<0}(R)=G\left(R\left[\frac{1}{t}\right]\right)$ for each $k$-algebra $R$.

We consider the cell $L_{G}^{\mu}=L_{G}^{+} t^{\mu} L_{G}^{+}$where $\mu$ is a non-negative coweight $\mu: \mathbb{G}_{m} \rightarrow T$ and the corresponding affine Schubert cell $\mathcal{Q}_{\mu}=\left(L_{G}^{+} t^{\mu} L_{G}^{+}\right) / L_{G}^{+}$of $\mathcal{Q}$. We know that $\mathcal{Q}_{\mu}$ is a smooth $k$-variety. Also the map $s_{0}: L_{G}^{+} \rightarrow G$ induces a $G$-equivariant map

$$
p_{\mu}: \mathcal{Q}_{\mu} \rightarrow G / P_{G}(-\mu)
$$

where $P_{G}(-\mu)$ stands for the Richardson parabolic subgroup attached to $\mu[19, \S 2]$. The orbit map $G \rightarrow \mathcal{Q}_{\mu}, g \mapsto g t^{\mu}$ induces a closed immersion $i_{\mu}: G / P_{G}(-\mu) \rightarrow \mathcal{Q}_{\mu}$ which is a section of $p_{\mu}$.

We consider the $k$-groups $L^{\geq \mu} G=t^{\mu} L_{G}^{+} t^{-\mu}$ and $L^{<\mu} G=t^{\mu} L_{G}^{<0} t^{-\mu}$. Let $J=$ $L_{G}^{+} \cap s_{0}^{-1}(B)$ and define $J \geq \mu=J \cap L_{G}^{\geq \mu}$ and $J^{\mu}=J \cap L_{G}^{<\mu}$.

According to [19, lemme 2.2], we have an isomorphism $J^{\mu} \times J \geq \mu \stackrel{\sim}{\longrightarrow} J$ and the map $J^{\mu} \rightarrow \mathcal{Q}_{\mu}, g \rightarrow g t^{\mu}$ is an open immersion; we denote by $\Omega^{\mu}$ its image. We choose a total order on $\Phi_{+}$; furthermore the product in $L_{G}^{+}$induces an isomorphism (of $k$-varieties) 


$$
j_{\mu}: \quad \prod_{\alpha \in \Phi \mid\langle\mu, \alpha\rangle \geq 1} \prod_{i=0}^{\langle\mu, \alpha\rangle} U_{\alpha, i} \stackrel{\sim}{\longrightarrow} \quad J^{\mu}
$$

where $U_{\alpha, i}$ is the image of $u_{\alpha, i}: \mathbb{G}_{a} \rightarrow L_{G}^{+}, x \mapsto u_{\alpha}\left(t^{i} x\right)$.

Lemma 9.5. The following diagram

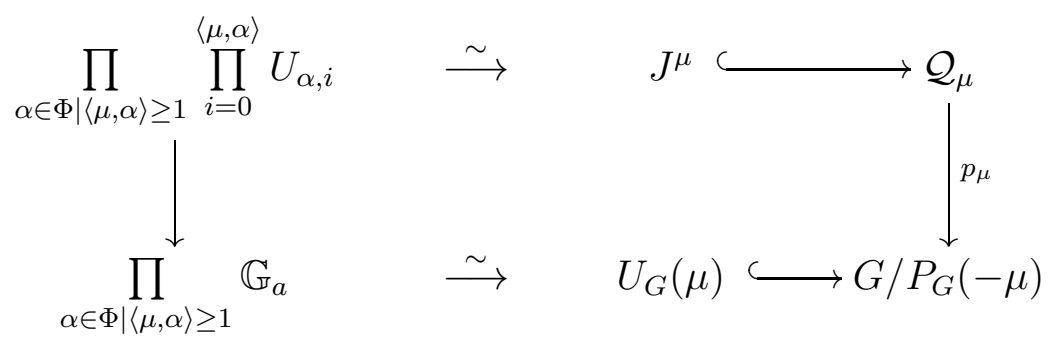

commutes and is cartesian where the bottom map is induced by the embedding $U_{G}(\mu) \hookrightarrow$ $G$ and the left vertical map is the projection on the factors involving $i=0$.

Note that $U_{G}(\mu) \rightarrow G / P_{G}(-\mu)$ is an open embedding (Bruhat big cell).

Proof. The commutativity is obvious. We have the the inclusions $\Omega_{\mu} \subseteq p_{\mu}^{-1}\left(U_{G}(\mu)\right.$.[1]) $\subset$ $\mathcal{Q}_{\mu}$ and we have to show that $\Omega_{\mu} \subseteq p_{\mu}^{-1}\left(U_{G}(\mu) \cdot[1]\right)$.

Up to extend $k$ to its algebraic closure, it enough to show that $p_{\mu}^{-1}\left(U_{G}(\mu) \cdot[1]\right)(k) \subseteq$ $\Omega_{\mu}(k)$. We are given an element $g . t^{\mu} \in p_{\mu}^{-1}\left(U_{G}(\mu)\right.$.[1])(k) with $g \in L_{G}^{+}(k)$. Since $g(0)$.[1] belongs in the big Bruhat cell $V=U_{G}(\mu)$. [1] of $G / P_{G}(-\mu)$, it follows that $g .[1] \in V(k[[t]])$. We get that $g \in U_{G}(\mu)(k[[t]]) P_{G}(-\mu)(k[[t]])$. We can assume that $g \in U_{G}(\mu)(k[[t]])=L_{U_{G}(\mu)}^{+}(k)$. The decomposition $J^{\mu} \times J \geq \mu \stackrel{\sim}{\longrightarrow} J$ induces a decomposition $J^{\mu} \times\left(J^{\geq \mu} \cap L_{U_{G}(\mu)}^{+}\right) \stackrel{\sim}{\longrightarrow} L_{U_{G}(\mu)}^{+}$. Since $J^{\geq \mu}$ fixes $t^{\mu}$, we get that $g . t^{\lambda} \in \Omega_{\mu}(k)$.

We get then an an isomorphism

$$
J_{+}^{\mu}:=\prod_{\alpha \in \Phi \mid\langle\mu, \alpha\rangle \geq 1} \prod_{i=1}^{\langle\mu, \alpha\rangle} U_{\alpha, i} \stackrel{\sim}{\longrightarrow} \quad p_{\mu}^{-1}([1]) \subset \mathcal{Q}_{\mu} .
$$

We put $E^{\mu}=p_{\mu}^{-1}([1])$, it is an affine $k$-space equipped with a left action of $P_{G}(-\mu)$. We get then a $P_{G}(-\mu)$-equivariant map $G \times{ }_{k} E^{\mu} \rightarrow \mathcal{Q}_{\mu}$ where $G$ is equipped with the left action provided by right translations. The next statement is well-known.

Lemma 9.6. The quotient $G \wedge^{P_{G}(-\mu)} E^{\mu}$ is representable by the smooth $k$-variety $\mathcal{Q}_{\mu}$.

Proof. The action $P_{G}(-\mu)$ on $G \times E^{\mu}$ (and on $G$ ) is free. We apply Lemma 10.2 to the $P_{G}(-\mu)$-morphism $G \times{ }_{k} E^{\mu} \rightarrow G$ and get that the fppf quotient $G \wedge^{P_{G}(-\mu)} E^{\mu}$ is representable by a $k$-scheme; we observe that this $k$-scheme is of finite type according 
to the permanence properties $\left[12,{ }_{2} .2 .7 .1\right]$. Similarly, using $\left[12,{ }_{4} .17 .7 .3\right]$, we see that $G \wedge{ }^{P_{G}(-\mu)} E^{\mu}$ is smooth over $G / P_{G}(-\mu)$.

By construction, the map $G / P_{G}(-\mu)$-map $G \wedge^{P_{G}(-\mu)} E^{\mu} \rightarrow \mathcal{Q}_{\mu}$ is an isomorphism over all geometric fibers over $G / P_{G}(-\mu)$. According to Grothendieck's fiberwise isomorphism criterion $\left[12,{ }_{4} \cdot 17.9 .5\right]$, we conclude that $Z \rightarrow \mathcal{Q}_{\mu}$ is an isomorphism.

Remarks 9.7. (a) If $\mu$ is minuscule, then the map $p_{\mu}$ is an isomorphism.

(b) If $\mu$ is quasi-minuscule (i.e. minimal but not minuscule), $p_{\mu}$ is the line bundle of Ngô-Polo [19, §7].

Proposition 9.8. [24, after 2.1.11] The morphism $i_{\mu}$ induces an isomorphism $G / P_{G}(-\mu) \stackrel{\sim}{\longrightarrow}{ }^{\sharp} \mathcal{Q}_{\mu}$ on the fixed point locus for the rotation action.

Proof. Since $\mathcal{Q}_{\mu}$ is smooth, the $k$-subvariety ${ }^{\sharp} \mathcal{Q}_{\mu}$ is smooth as well. It is then enough to check that $i_{\mu}$ induces an isomorphism $\left(G / P_{G}(-\mu)\right)(\bar{k}) \stackrel{\sim}{\longrightarrow}{ }^{\sharp} \mathcal{Q}_{\mu}(\bar{k})$. Let $q \in{ }^{\sharp} \mathcal{Q}_{\mu}(\bar{k})$. Up to conjugate by an element of $G(k)$ we can assume that $p_{\mu}(x)=1$ so that $x \in$ $E^{\mu}(k)$. But ${ }^{\sharp} E_{\mu}(k)=\left\{p_{\mu}(0)\right\}$ so that $x=i_{\mu}([1])$.

Taking into account Lemma 9.3 we get an isomorphism $G / P_{G}(-\mu) \stackrel{\sim}{\longrightarrow}{ }^{0} \mathcal{Q}_{\mu}$.

Proposition 9.9. Assume that $\mu>0$. Let $g \in L_{G}^{\mu}(R)$ where $R$ is a k-algebra.

(1) If $R$ is semilocal, then $r(g)=0$ if and only if $g \in G(R) t^{\mu} L_{G}^{+}(R)$.

(2) Assume that $r(g)=0$ and denote by $x$ the image of $g$ in $G / P_{G}(-\mu)(R) \stackrel{\sim}{\longrightarrow}$ ${ }^{0} \mathcal{Q}_{\mu}(R)$. It defines a $P_{G}(-\mu)$-torsor $E(g)$. Then $E(g)$ is a trivial $P_{G}(-\mu)$-torsor if and only if $g \in G(R) t^{\mu} L_{G}^{+}(R)$.

It follows of (1) that the strata $\mathcal{Q}_{\mu}$ which contains $[g]$ is encoded in $\operatorname{res}(g)$.

Proof. (1) If $g \in G(R) t^{\mu} L_{G}^{+}(R)$, then $r(g)=r\left(t^{\mu}\right)=0$. Conversely we assume that $r(g)=0$. We denote by $x \in{ }^{0} \mathcal{Q}_{\mu}(R)$ the image of $g$. Since $G / P_{G}(-\mu) \stackrel{\sim}{\longrightarrow}{ }^{0} \mathcal{Q}_{\mu}$ and $R$ is semilocal there exists $h \in G(R)$ such that $x=\left[h . t^{\mu}\right]$ [22, XXVI.5.10.(i)]. We conclude that $g \in G(R) t^{\mu} L_{G}(R)$.

(2) If $g=h t^{\mu} h^{\prime} \in G(R) t^{\mu} L_{G}^{+}(R)$, then $x=[h] \in\left(G / P_{G}(-\mu)\right)(R)$. We have $E(g)(R) \neq \emptyset$ so that the $P_{G}(-\mu)$-torsor $E(g)$ is trivial. Conversely we assume that $E(g)$ is the trivial $P_{G}(-\mu)$-torsor. Then $E(g)(R) \neq \emptyset$, that is there exists $h \in G(R)$ such that $x=[h]$. It follows that $h t^{\mu}=g \in L_{G}(R) / L_{G}^{+}(R)$ whence $g \in G(R) t^{\mu} L_{G}^{+}(R)$.

Proposition 9.10. (1) The ind $k$-variety ${ }^{\sharp} \mathcal{Q}$ is a $k$-scheme and we have ${ }^{\sharp} \mathcal{Q}=\bigsqcup_{\mu \geq 0}{ }^{0} \mathcal{Q}{ }_{\mu}$.

(2) Let $R$ be a semi-local connected $k$-algebra. We have

$$
{ }^{0} L_{G}(R)=\bigsqcup_{\mu \geq 0} G(R) t^{\mu} L_{G}^{+}(R) .
$$


Proof. (1) We write $\mathcal{Q}=\lim _{\theta \geq 0} \overline{\mathcal{Q}}_{\theta}$ as an inductive limit of projective varieties. Let $\theta$ be a non-negative coweight. The $\mathcal{Q}_{\mu}$ 's for $0 \leq \mu \leq \theta$ provide a stratification of $\overline{\mathcal{Q}}_{\theta}$.

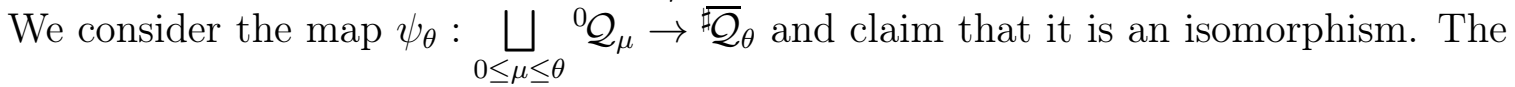
left-hand side is a projective variety and so is the right handside. Each piece ${ }^{0} \mathcal{Q}_{\mu}$ is a closed (smooth) subvariety of $\overline{\mathcal{Q}}_{\theta}$. Also ${ }^{0} \mathcal{Q}_{\mu} \cap{ }^{0} \mathcal{Q}_{\mu^{\prime}}=\emptyset$ for $\mu^{\prime} \neq \mu$ since the residue encodes the strata. It follows that $\psi_{\theta}$ is a closed immersion. On the other hand, the

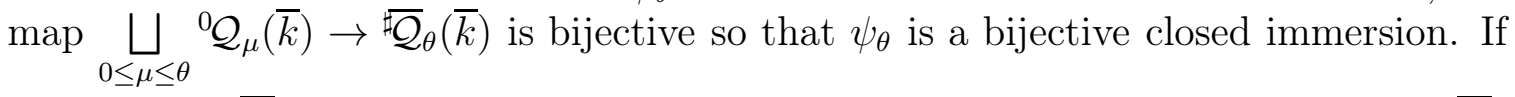
follows that $\AA_{\mathcal{Q}}=\bigsqcup_{0 \leq \mu \leq \theta} Z_{\theta, \mu}$ where $Z_{\theta, \mu}$ is the unique connected component of ${ }^{\sharp} \overline{\mathcal{Q}}_{\theta}$ containing the image of ${ }^{0} \mathcal{Q}_{\mu}$ for each $\mu$. We observe that $Z_{\theta, \theta}$ is closed in ${ }^{\sharp} \overline{\mathcal{Q}}_{\theta}$ and does not intersect the boundary ${ }^{\sharp} \overline{\mathcal{Q}}_{\theta} \backslash \mathcal{Q}_{\theta}$ so that ${ }^{\sharp} \mathcal{Q}_{\theta} \subseteq Z_{\theta, \theta} \subset \mathcal{Q}_{\theta}$. Thus ${ }^{\sharp} \mathcal{Q}_{\theta}=Z_{\theta, \mu}$ by taking the invariants under the rotation action.

We oberve that $Z_{\theta, \mu}=Z_{\theta^{\prime}, \mu}$ for all coweights satisfying $0 \leq \leq \theta \leq \theta^{\prime}$ so that ${ }^{\sharp} \mathcal{Q}_{\mu}=Z_{\theta, \mu}$ for all coweights satisfying $0 \leq \mu \leq \theta$. Thus $\psi_{\theta}$ is an isomorphism for all non-negative coweights $\theta$.

Passing to the limit on $\theta$ yieds the wished statement.

(2) We combine (1) and Proposition 9.9.

Given an element $g$ of $L_{G}^{\mu}(k)$, we would like to investigate in a few cases its index and its residue. We write it $g=g_{0}\left(\prod_{\alpha \in \Phi \mid\langle\mu, \alpha\rangle \geq 2} u_{\alpha}\left(\sum_{i=1}^{\langle\mu, \alpha\rangle-1} t^{i} x_{\alpha, i}\right)\right) t^{\mu}$. Then $g$ and $\left(\prod_{\alpha \in \Phi \mid\langle\mu, \alpha\rangle \geq 2} u_{\alpha}\left(\sum_{i=1}^{\langle\mu, \alpha\rangle-1} t^{i} x_{\alpha, i}\right)\right) t^{\mu}$ have same index and conjugated residues, so that we may work with $g=\left(\prod_{\alpha \in \Phi \mid\langle\mu, \alpha\rangle \geq 2} u_{\alpha}\left(\sum_{i=1}^{\langle\mu, \alpha\rangle-1} t^{i} x_{\alpha, i}\right)\right) t^{\mu}$.

The minuscule case. We have $g=t^{\mu}$ hence $r(g)=0$ and $\operatorname{res}(g)=t^{\mu}$.

The quasi-minuscule case. Let $\gamma$ be the unique root satisfying $\langle\mu, \gamma\rangle \geq 2$. We have $g=u_{\gamma}(t x) t^{\mu}$ for some $x \in k$. For $r=m / n$, it follows that

$$
\begin{aligned}
g^{-1} \sigma_{r}(g) & =t^{-\mu} u_{\gamma}(-t x) u_{\gamma}\left(t\left(1+u t^{r}\right) x\right) t^{\mu}\left(\sigma_{r}(t) / t\right)^{\mu} \\
& =t^{-\mu} u_{\gamma}\left(u t^{r+1} x\right) t^{-\mu}\left(\sigma_{r}(t) / t\right)^{\mu} \\
& =u_{\gamma}\left(u t^{r+1-\langle\mu, \gamma\rangle} x\right) t^{-\mu}\left(\sigma_{r}(t) / t\right)^{\mu} .
\end{aligned}
$$

It follows that $r=\langle\mu, \gamma\rangle-1$ if $x \neq 0$.

The rank one case. There exists a unique root $\alpha$ satisfying $\langle\mu, \alpha\rangle \geq 1$ and we assume that $\langle\mu, \alpha\rangle \geq 2$ (since the case $\langle\mu, \alpha\rangle=1$ is minuscule). We have $g=(P(t)) t^{\mu}$ for some polynomial $P \in k[t]$ of degree $\leq\langle\mu, \alpha\rangle-1$ satisfying $P(0)=0$. For $r=m / n$, 
it follows that

$g^{-1} \sigma_{r}(g)=t^{-\mu} u_{\alpha}(-P(t)) u_{\alpha}\left(P\left(t\left(1+u t^{r}\right)\right) t^{\mu}\left(\sigma_{r}(t) / t\right)^{\mu}=u_{\alpha}\left(\frac{P(t)-P\left(t\left(1+u t^{r}\right)\right)}{t^{\langle\mu, \alpha\rangle}}\right)\left(\sigma_{r}(t) / t\right)^{\mu}\right.$.

It follows that

$$
r \geq r(g) \Longleftrightarrow \frac{P(t)-P\left(t\left(1+u t^{r}\right)\right)}{t^{\langle\mu, \alpha\rangle}} \in k^{u}\left[\left[t^{1 / n}\right]\right] .
$$

For example, for $P(t)=t$, we find that $r(g)=\langle\mu, \alpha\rangle$ and that $\operatorname{res}(g)=u_{\alpha}$.

Corollary 9.11. With the preceding notations we have $r(g)=0$ if and only if $P=0$.

Note that this is coherent with Proposition 9.9.

Proof. If $P=0$, we have $g=t^{\mu}$ so that $r(g)=0$. Conversely, we assume that $r(g)=0$ so that $g^{-1} g(\lambda t) \in G\left(k\left[\lambda^{ \pm 1}\right][[t]]\right)$. The above computation with $\lambda=1+u t^{0}$ shows that

$$
g^{-1} g(\lambda t)=u_{\alpha}\left(\frac{P(t)-P(\lambda t)}{t^{\langle\mu, \alpha\rangle}}\right) \mu(\lambda) \in G\left(k\left[\lambda^{ \pm 1}\right][[t]]\right)
$$

Since $P$ is of degree $\langle\langle\mu, \alpha\rangle$, we conclude that $P=0$.

\section{APPENDIX: DESCENT}

Lemma 10.1. Let $S$ be a scheme. Let $G$ be a $S$-group scheme flat of finite type. We are given a left action of $G$ on an $S$-scheme $X$ of finite type such that $X$ admits a $G$-linearized line bundle $\mathcal{L}$ which is relatively ample over $S$. Let $T$ be an $S$-scheme and let $f: E \rightarrow T$ be a $G_{T}$-torsor. Then the contracted product $E \wedge^{G} X_{T}$ over $T$ is representable by a $T$-scheme. Furthermore we have

(i) the line bundle $\mathcal{M}=E \wedge^{G} \mathcal{L}$ on $E \wedge^{G} X_{T}$ is relatively ample over $T$.

(ii) If a $S$-group scheme $J$ acts (on the left) on $f: E \rightarrow T$ such that $T$ admits a $J$-linearized line bundle which is relatively ample over $S$, then $E \wedge^{G} X_{T}$ admits a J-linearized line bundle which is relatively ample over $T$.

Proof. The first part with property (i) is $[2, \S 10.2$, lemme 6]. For establishig (ii), we are given a $J$-linearized line bundle $\mathcal{N}_{0}$ on $T$ which is relatively ample over $S$. We consider the mapping $h: E \wedge^{G} X_{T} \rightarrow T$, we know that there exists a positive integer $n$ such that the line bundle $\mathcal{N}=h^{*}\left(\mathcal{N}_{0}\right)^{\otimes n} \otimes \mathcal{M}$ on $E \wedge^{G} X_{T} \rightarrow T$ is relatively ample over $T[11,4.6 .13$.(ii)]. This line bundle is $J$-linearized as desired.

Lemma 10.2. Let $S$ be a scheme and let $G$ be a flat $S$-group scheme locally of finite type. We are given a $G$-morphism of $S$-schemes $f: X \rightarrow Y$. We assume that $G$ acts freely on $X$ and on $Y$, that $f$ is affine and that the fppf quotient $Y / G$ is representable by a $S$-scheme. Then the fppf quotient $X / G$ is representable by an $S$-scheme. 
Proof. Put $Z:=Y / G$. Assume first that the $G$-torsor $Y \longrightarrow Z$ is trivial. Equivalently, the $G$-scheme $Y$ is isomorphic, over $S$, to $G \times{ }_{S} Z$. Choosing such an isomorphism allows to consider $f$ as a $G$-morphism $X \longrightarrow G \times_{S} Z$. Put $X_{0}:=f^{-1}\left(e \times_{S} Z\right)$; it is a closed subscheme of $X$, which is transverse to the $G$-action: the natural $G$ morphism $G \times{ }_{S} X_{0} \longrightarrow X$ is an isomorphism. Thus, $X / G$ is represented by $X_{0}$.

For the general case, we proceed by descent. Considering $f$ as a morphism of $Z$ schemes, we may replace $S$ by $Z$ (and $G$ by $G \times{ }_{S} Z$ ), and assume for simplicity that $Z=S$. Hence, $Y$ is a $G$-torsor over $S$. Put $S^{\prime}:=Y$. We are going to base-change the situation via the morphism $S^{\prime} \longrightarrow S$. Put

$$
f^{\prime}:=f \times_{S} S^{\prime}: X^{\prime}:=X \times_{S} S^{\prime} \longrightarrow Y^{\prime}:=Y \times_{S} S^{\prime} .
$$

Now, the $G$-torsor $Y^{\prime} \longrightarrow S^{\prime}$ is trivial, so that the quotient $X^{\prime} \longrightarrow X^{\prime} / G$ exists (and is a trivial $G$-torsor) by the discussion above. Since $X^{\prime} \longrightarrow Y^{\prime}$ is affine, $X^{\prime} / G \longrightarrow$ $Y^{\prime} / G=S^{\prime}$ is affine as well. It is equipped with a canonical descent data for the fpqc morphism $S^{\prime} \longrightarrow S$. Hence, this data is effective (by fpqc descent for affine schemes), yielding an arrow $\tilde{X} \longrightarrow S$. Now, using descent for morphisms, the $S^{\prime}$-arrow $X^{\prime} \longrightarrow X^{\prime} / G$ descends to an $S$-arrow $X \longrightarrow \tilde{X}$. This is the quotient $X \longrightarrow X / G$ we sought for. This fact can, again, be checked by descent.

\section{REFERENCES}

[1] A. Borel, J. Tits, Homomorphismes "abstraits" de groupes algébriques simples, Ann. of Math. 97 (1973), 499-571.

[2] S. Bosch, W. Lütkebohmert, M. Raynaud, Néron models, Ergebnisse der Mathematik und ihrer Grenzgebiete 21 (1990), Springer.

[3] A. Bouthier, K. Cesnavičius, Torsors on loop groups and the Hitchin fibration, to appear in Annales de l'Ecole normale supérieure.

[4] F. Bruhat, J. Tits, Groupes algébriques sur un corps local II. Existence d'une donnée radicielle valuée, Pub. Math. IHES 60 (1984), 5-184.

[5] J.-L. Colliot-Thélène, M. Ojanguren, Espaces principaux homogènes localement triviaux, Publications Mathématiques de l'IHES 75 (1992), 97-122.

[6] J.-L. Colliot-Thélène and J.-J. Sansuc, Principal homogeneous spaces under flasque tori: applications, J. Algebra 106 (1987), 148-205.

[7] B. Conrad, O. Gabber, G. Prasad, Pseudo-reductive groups, Cambridge University Press, second edition (2016).

[8] B. Conrad, The structure of solvable groups over general fields, Autour des schémas en groupes. Vol. II, 159-192, Panor. Synthèses 46 (2015), Soc. Math. France, Paris.

[9] C. Demarche, Cohomologie de Hochschild non abélienne et extensions de faisceaux en groupes, Autour des schémas en groupes, volume II, Panoramas et Synthèses 46, 255-292 (2015).

[10] M. Demazure, P. Gabriel, Groupes algébriques, North-Holland (1970).

[11] A. Grothendieck (avec la collaboration de J. Dieudonné), Eléments de Géométrie Algébrique II, Publications mathématiques de l'I.H.É.S. no 8 (1961).

[12] A. Grothendieck (avec la collaboration de J. Dieudonné), Eléments de Géométrie Algébrique $I V$, Publications mathématiques de l'I.H.É.S. no 20, 24, 28 and 32 (1964 - 1967). 
[13] M. Florence, Points rationnels sur les espaces homogènes et leurs compactifications, Transformation Groups 11 (2006), 161-176.

[14] O. Gabber, P. Gille, L. Moret-Bailly, Fibrés principaux sur les corps valués henséliens, Algebraic Geometry 5 (2014), 573-612.

[15] P. Gille, Torseurs sur la droite affine et R-équivalence, thèse (Orsay, 1994), author's URL.

[16] J. Giraud, Cohomologie non-abélienne, Springer (1970).

[17] U. Görtz, Affine Springer fibers and affine Deligne-Lusztig varieties, Affine flag manifolds and principal bundles, 1-50, Trends Math., Birkhäuser/Springer Basel AG, Basel, 2010.

[18] N. Guo, The Grothendieck-Serre Conjecture over Semilocal Dedekind Rings, to appear in Transformation Groups.

[19] B. C. Ngô, P. Polo, Résolutions de Demazure affines et formule de Casselman-Shalika géométrique, J. Algebraic Geom. 10 (2001), 515-547.

[20] O. Gabber, On pseudo-reductive groups and compactification theorems, Oberwolfach Reports (2012), 2371-2374.

[21] G. Prasad, Elementary proof of a theorem of Bruhat-Tits-Rousseau and of a theorem of Tits, Bull. Soc. Math. France 110 (1982), 197-202.

[22] Séminaire de Géométrie algébrique de l'I. H. E. S., 1963-1964, schémas en groupes, dirigé par M. Demazure et A. Grothendieck, Lecture Notes in Math. 151-153. Springer (1970).

[23] Stacks project, https://stacks.math.columbia.edu

[24] X. Zhu, An introduction to affine Grassmannians and the geometric Satake equivalence, Geometry of moduli spaces and representation theory, 59-154, IAS/Park City Math. Ser., 24, Amer. Math. Soc., Providence, RI, 2017.

Institut de Mathématiques de Jussieu, Université Paris 6, Place Jussieu, 75005 PARIS, France

Email address: mathieu.florence@imj-prg.fr

UMR 5208 Institut Camille Jordan - Université Claude Bernard Lyon 143 BouleVARD DU 11 nOVEmbre 1918, 69622 Villeurbanne CEDEX - France

Email address: gille@math.univ-lyon1.fr 